\title{
Efficient Compensation of Transmitter and Receiver IQ Imbalance in OFDM Systems
}

\author{
Deepaknath Tandur and Marc Moonen (EURASIP Member) \\ K. U. Leuven, ESAT/SCD-SISTA, Kasteelpark Arenberg 10, 3001 Leuven-Heverlee, Belgium \\ Correspondence should be addressed to Deepaknath Tandur, deepaknath.tandur@esat.kuleuven.be
}

Received 1 December 2009; Revised 21 June 2010; Accepted 3 August 2010

Academic Editor: Ana Pérez-Neira

Copyright ( $) 2010$ D. Tandur and M. Moonen. This is an open access article distributed under the Creative Commons Attribution License, which permits unrestricted use, distribution, and reproduction in any medium, provided the original work is properly cited.

Radio frequency impairments such as in-phase/quadrature-phase (IQ) imbalances can result in a severe performance degradation in direct-conversion architecture-based communication systems. In this paper, we consider the case of transmitter and receiver IQ imbalance together with frequency selective channel distortion. The proposed training-based schemes can decouple the compensation of transmitter and receiver IQ imbalance from the compensation of channel distortion in an orthogonal frequency division multiplexing (OFDM) systems. The presence of frequency selective channel fading is a requirement for the estimation of IQ imbalance parameters when both transmitter/receiver IQ imbalance are present. However, the proposed schemes are equally applicable over a frequency flat/frequency selective channel when either transmitter or only receiver IQ imbalance is present. Once the transmitter and receiver IQ imbalance parameters are estimated, a standard channel equalizer can be applied to estimate/compensate for the channel distortion. The proposed schemes result in an overall lower training overhead and a lower computational requirement, compared to the joint compensation of transmitter/receiver IQ imbalance and channel distortion. Simulation results demonstrate that the proposed schemes provide a very efficient compensation with performance close to the ideal case without any IQ imbalance.

\section{Introduction}

Multicarrier modulation techniques such as orthogonal frequency division multiplexing (OFDM) are widely adopted transmission techniques for broadband communication systems [1]. OFDM has been adopted in a variety of wireless communication standards, for example, for wireless local area networks (WLANs) [2], wireless metropolitan area network (WiMAX) [3], and digital video broadcasting (DVB-T) [4]. The direct-conversion (or zero IF) architecture is an attractive front-end architecture for such systems [5]. Direct-conversion front-end architectures are typically small in size and can be easily integrated on a single chip, unlike the traditional superheterodyne architecture. These frontends also provide a high degree of flexibility in supporting a growing number of wireless standards as required in today's communication systems. However, direct-conversion frontends can be very sensitive to analog imperfections, especially when low-cost components are used in the manufacturing process. These front-end imperfections can result in radio frequency (RF) impairments such as in-phase/quadraturephase (IQ) imbalance. The IQ imbalance can result in a severe performance degradation, rendering the communication system inefficient or even useless. Rather than reducing the IQ imbalance by increasing the design time and the component cost, it is easier and more flexible to tolerate the IQ imbalance in the analog domain and then compensate for it digitally.

The effects of IQ imbalance have been studied and compensation schemes for OFDM systems have been developed in [6-20]. In [7-10], efficient digital compensation schemes have been developed for the case of receiver IQ imbalance together with carrier frequency offset (CFO). In $[11,12]$, these problems have been extended to also consider transmitter IQ imbalance together with receiver IQ imbalance and CFO. However, all these works consider only the effects of frequency independent IQ imbalance. For wideband communication systems it is important to also consider frequency selective distortions introduced by IQ imbalances. These frequency selective distortions arise 
mainly due to mismatched filters in the I and Q branch of the front-end. In [13, 14], efficient blind compensation schemes for frequency selective receiver IQ Imbalance have been developed. Recently in [15], a compensation scheme has been proposed that can decouple the frequency selective receiver IQ imbalance from the channel distortion, resulting in a reliable compensation with a small training overhead. In [16-18], joint compensation of frequency selective transmitter and receiver IQ imbalance has been considered with residual $\mathrm{CFO}$, no $\mathrm{CFO}$ and under high mobility conditions respectively. In [19], we have proposed a generally applicable adaptive frequency domain equalizer for the joint compensation of frequency selective transmitter/receiver IQ imbalance and channel distortion, for the case of an insufficient cyclic prefix (CP) length. The overall equalizer is based on a so-called per-tone equalization (PTEQ) [21]. In [20], we have proposed a low-training overhead equalizer for the general case of frequency selective transmitter and receiver IQ imbalance together with $\mathrm{CFO}$ and channel distortion for single-input single-output (SISO) systems. However, the proposed scheme cannot decouple the transmitter/receiver IQ imbalance from the channel distortion when there is no CFO.

In this paper, we consider the case of transmitter and receiver IQ imbalance together with frequency selective channel distortion. We propose estimation/compensation schemes that can decouple the compensation of transmitter and receiver IQ imbalance from the compensation of channel distortion. The proposed schemes require the presence of frequency selective channel fading for the estimation of IQ imbalance parameters when both transmitter/receiver IQ imbalance are present. However, the proposed schemes are equally applicable over a frequency flat/frequency selective channel when either transmitter or only receiver IQ imbalance is present. Once the transmitter and receiver IQ imbalance parameters are known, a standard channel equalizer requiring only one training symbol can be applied to estimate/compensate for the channel distortion. The proposed schemes result in an overall lower training overhead and a lower computational requirement, compared to the joint estimation/compensation scheme [11, 16-19]. It is to be noted that the proposed schemes do not take into account the effects of CFO. Since OFDM-based systems tend to be sensitive to CFO, there may be a need for additional fine synchronization of the carrier frequency on the analog side. A low-cost and low-training overhead transmitter/receiver IQ imbalance digital compensation scheme that is equally applicable with and without CFO, remains a challenge for future studies.

The paper is organized as follows. The input-output OFDM system model is presented in Section 2. Section 3 explains the IQ imbalance compensation scheme. Computer simulations are shown in Section 4 and finally the conclusion is given in Section 5.

Notation. Vectors are indicated in bold and scalar parameters in normal font. Superscripts \{\}$^{*},\{\}^{T},\{\}^{H}$ represent conjugate, transpose, and Hermitian transpose, respectively. $\mathbf{F}_{N}$ and $\mathbf{F}_{N}^{-1}$ represent the $N \times N$ discrete Fourier transform and its inverse. $\mathbf{I}_{N}$ is the $N \times N$ identity matrix and $\mathbf{0}_{M \times N}$ is the $M \times N$ all zero matrix. Operators!, · and $\div$ denote factorial component-wise vector multiplication and component-wise vector division, respectively. The operator $\star$ in the expression $\mathbf{c}=\mathbf{a} \star \mathbf{b}$ denotes a truncated linear convolution operation between the two vector sequences a and $\mathbf{b}$ of length $N_{a}$ and $N_{b}$, respectively. The vector sequence c is of length $N_{b}$ obtained by taking only the first $N_{b}$ elements out of the linear convolution operation that typically results in a sequence of length $N_{a}+N_{b}-1$.

\section{System Model}

Let $\mathbf{S}$ be an uncoded frequency domain OFDM symbol of size $(N \times 1)$ where $N$ is the number of tones. This symbol is transformed to the time domain by an inverse discrete Fourier transform (IDFT). A cyclic prefix (CP) of length $v$ is then added to the head of the symbol. The resulting time domain baseband symbol $\mathbf{s}$ is then given as

$$
\mathbf{s}=\mathbf{P}_{\mathrm{CI}} \mathbf{F}_{N}^{-1} \mathbf{S}
$$

where $\mathbf{P}_{\mathrm{CI}}$ is the $\mathrm{CP}$ insertion matrix given by

$$
\mathbf{P}_{\mathrm{CI}}=\left[\begin{array}{c|c}
\mathbf{0}_{(v \times N-v)} & \mathbf{I}_{v} \\
\hline \mathbf{I}_{N}
\end{array}\right] .
$$

The symbol $\mathbf{s}$ is parallel-to-serial converted before being fed to the transmitter front-end. Frequency selective (FS) IQ imbalance results from two mismatched front-end filters in the I and $\mathrm{Q}$ branches, with frequency responses given as $\mathbf{H}_{t i}=\mathbf{F}_{N} \mathbf{h}_{t i}$ and $\mathbf{H}_{t q}=\mathbf{F}_{N} \mathbf{h}_{t q}$, where $\mathbf{h}_{t i}$ and $\mathbf{h}_{t q}$ are the impulse response of the respective I and Q branch mismatched filters. Both $\mathbf{h}_{t i}$ and $\mathbf{h}_{t q}$ are considered to be $L_{t}$ long (and then possibly padded again with $N-L_{t}$ zero elements). The I and Q branch frequency responses $\mathbf{H}_{t i}$ and $\mathbf{H}_{t q}$ are of length $N$.

We represent the frequency independent (FI) IQ imbalance by an amplitude and phase mismatch $g_{t}$ and $\phi_{t}$ between the I and Q branches. Following the derivation in [13], the equivalent baseband symbol $\mathbf{p}$ of length $N+v$ after front-end distortions is given as

$$
\mathbf{p}=\mathbf{g}_{t a} \star \mathbf{s}+\mathbf{g}_{t b} \star \mathbf{s}^{*},
$$

where

$$
\begin{aligned}
& \mathbf{g}_{t a}=\mathbf{F}_{N}^{-1} \mathbf{G}_{t a}=\mathbf{F}_{N}^{-1}\left(\frac{\mathbf{H}_{t i}+g_{t} e^{-j \phi_{t}} \mathbf{H}_{t q}}{2}\right), \\
& \mathbf{g}_{t b}=\mathbf{F}_{N}^{-1} \mathbf{G}_{t b}=\mathbf{F}_{N}^{-1}\left(\frac{\mathbf{H}_{t i}-g_{t} e^{j \phi_{t}} \mathbf{H}_{t q}}{2}\right) .
\end{aligned}
$$

Here $\mathbf{g}_{t a}$ and $\mathbf{g}_{t b}$ are mostly truncated to length $L_{t}$ (and then possibly padded again with $N-L_{t}$ zero elements). They represent the combined FI and FS IQ imbalance at the transmitter. $\mathbf{G}_{t a}$ and $\mathbf{G}_{t b}$ are the frequency domain representations of $\mathbf{g}_{t a}$ and $\mathbf{g}_{t b}$, respectively. Both $\mathbf{G}_{t a}$ and $\mathbf{G}_{t b}$ are of length $N \cdot e^{j x}$ represents the exponential function on $x$ and $j=\sqrt{-1}$. 
An expression similar to (3) can be used to model IQ imbalance at the receiver. Let $\mathbf{z}$ represent the downconverted baseband complex symbol after being distorted by combined FS and FI receiver IQ imbalance. The overall receiver IQ imbalance is modelled by filters $\mathbf{g}_{r a}$ and $\mathbf{g}_{r b}$ of length $L_{r}$, where $\mathbf{g}_{r a}$ and $\mathbf{g}_{r b}$ are defined similar to $\mathbf{g}_{t a}$ and $\mathbf{g}_{t b}$ in (3). The received symbol $\mathbf{z}$ of length $N+v$ can then be written as

$$
\mathbf{z}=\mathbf{g}_{r a} \star \mathbf{r}+\mathbf{g}_{r b} \star \mathbf{r}^{*},
$$

where

$$
\mathbf{r}=\mathbf{c} \star \mathbf{p}+\mathbf{n} .
$$

Here, $\mathbf{r}$ is the received symbol before any receiver IQ imbalance distortion. $\mathbf{r}$ is of length $N+\nu, \mathbf{c}$ is the baseband equivalent of the multipath frequency selective quasistatic channel of length $L$, and $\mathbf{n}$ is the additive white Gaussian noise (AWGN). The channel is considered to be static for the duration of one entire packet consisting of training symbols followed by data symbols. Equation (3) can be substituted in (5) leading to

$$
\begin{aligned}
\mathbf{z}= & \left(\mathbf{g}_{r a} \star \mathbf{c} \star \mathbf{g}_{t a}+\mathbf{g}_{r b} \star \mathbf{c}^{*} \star \mathbf{g}_{t b}^{*}\right) \star \mathbf{s}+\mathbf{g}_{r a} \star \mathbf{n} \\
& +\left(\mathbf{g}_{r a} \star \mathbf{c} \star \mathbf{g}_{t b}+\mathbf{g}_{r b} \star \mathbf{c}^{*} \star \mathbf{g}_{t a}^{*}\right) \star \mathbf{s}^{*}+\mathbf{g}_{r b} \star \mathbf{n}^{*} \\
= & \mathbf{d}_{a} \star \mathbf{s}+\mathbf{d}_{b} \star \mathbf{s}^{*}+\mathbf{n}_{c},
\end{aligned}
$$

where $\mathbf{d}_{a}$ and $\mathbf{d}_{b}$ are the combined transmitter IQ imbalance, channel and receiver IQ imbalance impulse responses of length $L_{t}+L+L_{r}-2$, and $\mathbf{n}_{c}$ is the received noise modified by the receiver IQ imbalance.

The downconverted received symbol $\mathbf{z}$ is serial-toparallel converted and the part corresponding to the $\mathrm{CP}$ is removed. The resulting vector is then transformed to the frequency domain by the discrete Fourier transform (DFT) operation. In this paper, we assume the CP length $v$ to be larger than the length of $\mathbf{d}_{a}$ and $\mathbf{d}_{b}$, thus leading to no intersymbol interference (ISI) between the two consecutive OFDM symbols. The frequency domain received symbol $\mathbf{Z}$ of length $N$ can then be written as

$$
\begin{aligned}
\mathbf{Z}= & \mathbf{F}_{N} \mathbf{P}_{\mathbf{C R}}\{\mathbf{z}\} \\
= & \mathbf{D}_{a} \cdot \mathbf{S}+\mathbf{D}_{b} \cdot \mathbf{S}_{m}^{*}+\mathbf{N}_{c} \\
= & \left(\mathbf{G}_{r a} \cdot \mathbf{G}_{t a} \cdot \mathbf{C}+\mathbf{G}_{r b} \cdot \mathbf{G}_{t b_{m}}^{*} \cdot \mathbf{C}_{m}^{*}\right) \cdot \mathbf{S}+\mathbf{G}_{r a} \cdot \mathbf{N} \\
& +\left(\mathbf{G}_{r a} \cdot \mathbf{G}_{t b} \cdot \mathbf{C}+\mathbf{G}_{r b} \cdot \mathbf{G}_{t a_{m}}^{*} \cdot \mathbf{C}_{m}^{*}\right) \cdot \mathbf{S}_{m}^{*}+\mathbf{G}_{r b} \cdot \mathbf{N}_{m}^{*}
\end{aligned}
$$

where $\mathbf{P}_{\mathrm{CR}}$ is the $\mathrm{CP}$ removal matrix given as

$$
\mathbf{P}_{\mathrm{CR}}=\left[\mathbf{0}_{(N \times v)} \mid \mathbf{I}_{N}\right] \text {. }
$$

Here $\mathbf{G}_{r a}, \mathbf{G}_{r b}, \mathbf{C}, \mathbf{D}_{a}, \mathbf{D}_{b}, \mathbf{N}_{c}$, and $\mathbf{N}$ are of length $N$. They represent the frequency domain responses of $\mathbf{g}_{r a}, \mathbf{g}_{r b}, \mathbf{c}, \mathbf{d}_{a}, \mathbf{d}_{b}, \mathbf{n}_{c}$, and $\mathbf{n}$. The vector operator ()$_{m}$ denotes the mirroring operation in which the vector indices are reversed, such that $\mathbf{S}_{m}[l]=\mathbf{S}\left[l_{m}\right]$ where $l_{m}=2+N-l$ for $l=2 \cdots N$ and $l_{m}=l$ for $l=1$. Here $\mathbf{S}_{m}[l]$ represents the $l$ th element of $\mathbf{S}_{m}$.

Equation (8) shows that due to transmitter and receiver IQ imbalance, power leaks from the mirror carrier $\left(\mathbf{S}_{m}^{*}\right)$ into the carrier under consideration (S), that is, the imbalance causes intercarrier interference (ICI). Based on (8), the image rejection ratio (IRR) of the analog front-end processing for the tone $[l]$ can be defined as

$$
\operatorname{IRR}[l]=10 \log _{10} \frac{\left|\mathbf{D}_{a}[l]\right|^{2}}{\left|\mathbf{D}_{b}[l]\right|^{2}} .
$$

In practice, the IRR $[l]$ due to IQ imbalance is in the order of 20-40 dB for one terminal (transmitter or receiver) [22]. The joint effect of transmitter and receiver IQ imbalance is thus expected to be more severe. In Section 3, we propose efficient compensation schemes for an OFDM system impaired with transmitter and receiver IQ imbalance. The improvement in IRR performance in the presence of these compensation schemes is later discussed in Section 4.

\section{IQ Imbalance Compensation}

3.1. Joint Transmitter/Receiver IQ Imbalance and Channel Distortion Compensation. We first focus on the joint compensation of transmitter/receiver IQ imbalance and channel distortion. In the following Sections 3.2-3.4, we will develop more efficient decoupled compensation schemes.

Equation (8) can be rewritten for the received symbol $\mathbf{Z}$ and the complex conjugate of its mirror symbol $\mathbf{Z}_{m}^{*}$ as follows:

$$
\underbrace{\left[\begin{array}{c}
\mathbf{Z}[l] \\
\mathbf{Z}^{*}\left[l_{m}\right]
\end{array}\right]}_{\mathbf{Z}_{\text {tot }}[l]}=\underbrace{\left[\begin{array}{cc}
\mathbf{D}_{a}[l] & \mathbf{D}_{b}[l] \\
\mathbf{D}_{b}^{*}\left[l_{m}\right] & \mathbf{D}_{a}^{*}[l]
\end{array}\right]}_{\mathbf{D}_{\text {tot }}[l]} \underbrace{\left[\begin{array}{c}
\mathbf{S}[l] \\
\mathbf{S}^{*}\left[l_{m}\right]
\end{array}\right]}_{\mathbf{S}_{\text {tot }}[l]}+\left[\begin{array}{c}
\mathbf{N}_{c}[l] \\
\mathbf{N}_{c}^{*}\left[l_{m}\right]
\end{array}\right] .
$$

The matrix $\mathbf{D}_{\text {tot }}[l]$ represents the joint transmitter IQ imbalance, receiver IQ imbalance, and channel distortion for the received symbol matrix $\mathbf{Z}_{\mathrm{tot}}[l]$.

Assuming $\mathbf{D}_{\text {tot }}[l]$ is known, then a symbol estimate $\widetilde{\boldsymbol{S}}_{\text {tot }}[l]$ can be obtained based on zero forcing $(\mathrm{ZF})$ criterion:

$$
\tilde{\mathbf{S}}_{\text {tot }}[l]=\mathbf{D}_{\text {tot }}[l]^{-1} \mathbf{Z}_{\text {tot }}[l] .
$$

The $\mathbf{D}_{\text {tot }}[l]$ can be obtained with a training-based estimation scheme. We consider the availability of an $M_{l}$ long sequence of so-called long training symbols (LTS), all constructed based on (1). Equation (11) can then be used for all LTS as follows:

$$
\mathbf{Z}_{\text {tot- }}^{T r}[l]=\mathbf{D}_{\text {tot- }}[l] \mathbf{S}_{\text {tot }}^{T r}[l]+\left[\mathbf{N}_{c}^{(1)}[l] \cdots \mathbf{N}_{c}^{\left(M_{l}\right)}[l]\right],
$$

where $\mathbf{Z}_{\text {tot- }}^{T r}[l]=\left[\mathbf{Z}^{(1)}[l] \cdots \mathbf{Z}^{\left(M_{l}\right)}[l]\right], \mathbf{D}_{\text {tot- }}[l]=\left[\mathbf{D}_{a}[l] \mathbf{D}_{b}[l]\right]$, and

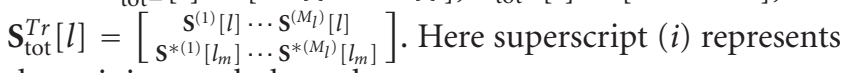
the training symbol number.

An estimate of $\mathbf{D}_{\text {tot- }}[l]$ can then be obtained as

$$
\widetilde{\mathbf{D}}_{\text {tot- }}[l]=\mathbf{S}_{\text {tot }}^{T r^{\dagger}}[l] \mathbf{Z}_{\text {tot }-}^{T r}[l],
$$




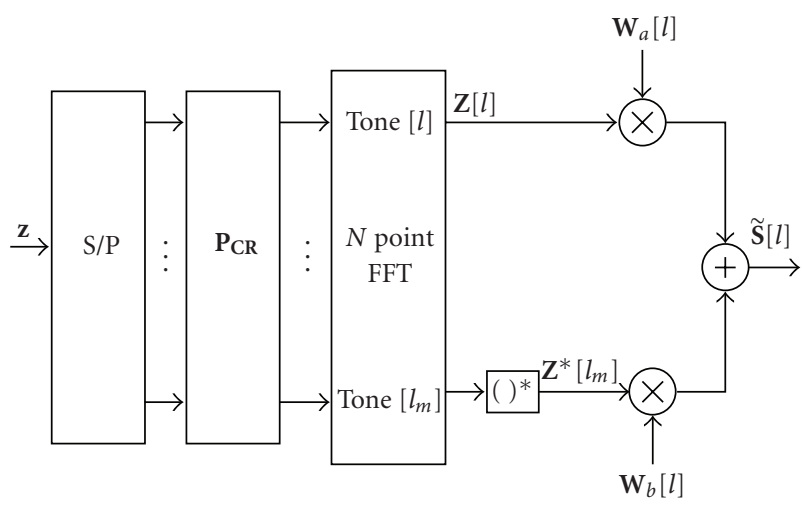

FIgURE 1: Joint compensation scheme for OFDM system in the presence of transmitter and receiver IQ imbalance.

where $\dagger$ is the pseudoinverse operation. Equation (13) represents $M_{l}$ equations in 2 unknowns. Hence to estimate $\mathbf{D}_{\text {tot- }}[l]$, we need the LTS sequence length $M_{l} \geq 2$. If only two LTS are available, that is, $M_{l}=2$, we can guarantee the invertibility $\boldsymbol{S}_{\mathrm{tot}}^{T r^{-1}}[l]$ by generating training symbols such that $\boldsymbol{S}^{*(2)}\left[l_{m}\right]=-\mathbf{S}^{(1)}[l]$. A longer training sequence will provide improved estimates due to a better noise averaging. Once $\widetilde{\mathbf{D}}_{\text {tot- }}[l]$ and hence $\widetilde{\mathbf{D}}_{\text {tot }}[l]$ is accurately known, we can obtain $\widetilde{\mathbf{S}}_{\mathrm{tot}}[l]$ as in (12). This is the principle behind the joint compensation scheme in $[11,17]$. It should be noted that (14) is also valid in the presence of either only transmitter IQ imbalance or only receiver IQ imbalance. In the absence of any IQ imbalance, the term $\mathbf{D}_{b}[l]=0$, a standard OFDM decoder, is then used to estimate the channel.

Based on (14), we can also directly generate symbol estimates as

$$
\tilde{\mathbf{S}}[l]=\left[\begin{array}{ll}
\mathbf{W}_{a}[l] & \mathbf{W}_{b}[l]
\end{array}\right]\left[\begin{array}{c}
\mathbf{Z}[l] \\
\mathbf{Z}^{*}\left[l_{m}\right]
\end{array}\right] .
$$

Here, $\mathbf{W}_{a}[l]$ and $\mathbf{W}_{b}[l]$ are the coefficients of a frequency domain equalizer (FEQ). The FEQ coefficients are estimated based on a mean square error (MSE) minimization:

$$
\min _{\mathbf{W}_{a}[l], \mathbf{W}_{b}[l]} \Xi\left\{\left|\tilde{\mathbf{S}}[l]-\left[\begin{array}{ll}
\mathbf{W}_{a}[l] & \mathbf{W}_{b}[l]
\end{array}\right]\left[\begin{array}{c}
\mathbf{Z}[l] \\
\mathbf{Z}^{*}\left[l_{m}\right]
\end{array}\right]\right|^{2}\right\} .
$$

The basic difference between the compensation in (12) and (15) is that (12) requires an estimate of the joint channel and transmitter/receiver IQ imbalance matrix $\mathbf{D}_{\text {tot }}[l]$, while (15) performs a direct equalization under noise. The FEQ coefficients can be obtained directly from the LTS based on a least squares (LS) or a recursive least squares (RLS) estimation scheme. The equalizer can subsequently be applied to data symbols as long as the channel characteristics do not change. The FEQ scheme is illustrated in Figure 1.

A disadvantage of this joint transmitter/receiver IQ imbalance and channel distortion compensation scheme is that $\mathbf{D}_{\text {tot }}[l]$ has to be reestimated for every variation of the channel characteristics even when the IQ imbalance parameters are constant. In the following sections, we develop a compensation scheme where the transmitter/receiver IQ imbalance can be decoupled from the channel distortion. This results in a compensation scheme where in time-varying scenarios only the channel parameters have to be reestimated while the IQ imbalance parameters are indeed kept constant. The decoupled scheme then in particular has a reduced training requirement. In Section 3.2, we develop a decoupled compensation scheme for the case of only transmitter IQ imbalance. This compensation scheme is then (Section 3.3) extended for a system impaired with both transmitter and receiver IQ imbalance.

3.2. Decoupled Transmitter IQ Imbalance and Channel Distortion Compensation. In the case of only transmitter IQ imbalance and no receiver IQ imbalance $\left(\mathbf{G}_{r a}[l]=\right.$ $1, \mathbf{G}_{r b}[l]=0$ ), we can decouple $\mathbf{D}_{\text {tot }}[l]$ as follows:

$$
\begin{aligned}
\mathbf{D}_{\text {tot }}[l] & =\left[\begin{array}{cc}
\mathbf{D}_{a}[l] & \mathbf{D}_{b}[l] \\
\mathbf{D}_{b}^{*}\left[l_{m}\right] & \mathbf{D}_{a}^{*}\left[l_{m}\right]
\end{array}\right] \\
& =\underbrace{\left[\begin{array}{cc}
\mathbf{B}[l] & 0 \\
0 & \mathbf{B}^{*}\left[l_{m}\right]
\end{array}\right]}_{\mathbf{B}_{\text {tot }}[l]} \underbrace{\left[\begin{array}{cc}
1 & \mathbf{Q}_{t}[l] \\
\mathbf{Q}_{t}^{*}\left[l_{m}\right] & 1
\end{array}\right],}_{\mathbf{Q}_{\text {tot }}[l]},
\end{aligned}
$$

where $\mathbf{Q}_{t}[l]=\mathbf{G}_{t b}[l] / \mathbf{G}_{t a}[l]$ is the transmitter IQ imbalance gain parameter and $\mathbf{B}[l]=\mathbf{G}_{t a}[l] \mathbf{C}[l]$ is a composite channel. The estimates $\widetilde{\mathbf{Q}}_{t}[l]$ and $\widetilde{\mathbf{B}}[l]$ of $\mathbf{Q}_{t}[l]$ and $\mathbf{B}[l]$ can be directly obtained from $\widetilde{\mathbf{D}}_{\text {tot- }}[l](14)$ as

$$
\begin{aligned}
\widetilde{\mathbf{Q}}_{t}[l] & =\frac{\widetilde{\mathbf{D}_{b}}[l]}{\widetilde{\mathbf{D}_{a}}[l]}, \\
\widetilde{\mathbf{B}}[l] & =\widetilde{\mathbf{D}}_{a}[l],
\end{aligned}
$$

where $\widetilde{\mathbf{D}}_{a}[l]$ and $\widetilde{\mathbf{D}}_{b}[l]$ are the estimates of $\mathbf{D}_{a}[l]$ and $\mathbf{D}_{b}[l]$. In the case of only FI transmitter IQ imbalance, $\widetilde{\mathbf{Q}}_{t}[l]$ can be averaged over all the tones to obtain an improved estimate $\widetilde{\mathrm{Q}}_{t}=1 / N \sum_{l=1}^{N} \widetilde{\mathbf{Q}}_{t}[l]$.

Once $\widetilde{\mathbf{Q}}_{t}[l]$ is available, variations in channel can be tracked by reestimating $\widetilde{\mathbf{B}}[l]$ with

$$
\widetilde{\mathbf{B}}[l]=\frac{\mathbf{Z}[l]}{\mathbf{S}[l]+\widetilde{\mathbf{Q}}_{t}[l] \mathbf{S}^{*}\left[l_{m}\right]} .
$$

Only one training symbol is required to reestimate $\widetilde{\mathbf{B}}[l]$. A longer training sequence will provide improved estimates.

During the compensation phase, the $\widetilde{\mathbf{D}}_{\text {tot }}[l]$ can once again be formulated from the new composite channel estimate $\widetilde{\mathbf{B}}[l]$ and the transmitter IQ imbalance gain parameter $\widetilde{\mathbf{Q}}_{t}[l]$. We can now obtain the estimate of the transmitted OFDM symbol by the following equation:

$$
\widetilde{\mathbf{S}}_{\text {tot }}[l]=\underbrace{\left(\widetilde{\mathbf{B}}_{\text {tot }}[l] \widetilde{\mathbf{Q}}_{\text {tot }}[l]\right)^{-1}}_{\widetilde{\mathbf{D}}_{\text {tot }}[l]} \mathbf{Z}_{\text {tot }}[l],
$$

where $\widetilde{\mathbf{Q}}_{t_{\text {tot }}}[l]$ and $\widetilde{\mathbf{B}}_{\text {tot }}[l]$ are the estimates of $\mathbf{Q}_{t_{\text {tot }}}[l]$ and $\mathbf{B}_{\text {tot }}[l]$. We will refer to the proposed decoupled based frequency domain estimation/compensation scheme (18)(20) as D-FEQ. 
Predistortion of Transmitted Symbols. The D-FEQ compensation scheme based on (20) performs the compensation of transmitter IQ imbalance at the receiver. As the joint channel distortion and transmitter IQ imbalance compensation is based on a zero forcing equalization, the compensation may be affected by noise enhancement, especially so in poor SNR conditions. An alternative solution, to avoid the noise enhancement, is to compensate for the transmitter IQ imbalance already at the transmitter. This can be obtained by distorting the transmitted symbol before the IDFT operation such that the resulting transmitted symbol is free of any transmitter IQ imbalance. The predistortion scheme provides better performance as in this case the receiver only has to equalize the channel with a very short training overhead. The transmitted symbol recovery can then be obtained based on an MMSE or ZF equalization scheme at the receiver. A predistortion system requires a feedback mechanism between the receiver and the transmitter, as will be explained next.

In the predistortion scheme, the new OFDM symbol $\mathbf{S}_{\mathbf{n}}$ is defined as $\mathbf{S}_{\mathbf{n}}=\mathbf{S}-\widetilde{\mathbf{Q}}_{t} \cdot \mathbf{S}_{m}^{*}$ where $\widetilde{\mathbf{Q}}_{t}$ is the $\mathbf{Q}_{t}$ estimate fed back from the receiver. In matrix form, $\mathbf{S}_{\mathbf{n}}[l]$ and $\mathbf{S}_{\mathbf{n}}^{*}\left[l_{m}\right]$ can be written as

$$
\left[\begin{array}{c}
\mathbf{S}_{\mathbf{n}}[l] \\
\mathbf{S}_{\mathbf{n}}^{*}\left[l_{m}\right]
\end{array}\right]=\left[\begin{array}{cc}
1 & -\widetilde{\mathbf{Q}}_{t}[l] \\
-\widetilde{\mathbf{Q}}_{t}^{*}\left[l_{m}\right] & 1
\end{array}\right]\left[\begin{array}{c}
\mathbf{S}[l] \\
\mathbf{S}^{*}\left[l_{m}\right]
\end{array}\right]
$$

Now (11) is modified as,

$$
\begin{aligned}
& \mathbf{Z}_{\text {tot }}[l] \\
& =\left[\begin{array}{cc}
\mathbf{B}[l] & 0 \\
0 & \mathbf{B}^{*}\left[l_{m}\right]
\end{array}\right]\left[\begin{array}{cc}
1 & \mathbf{Q}_{t}[l] \\
\mathbf{Q}_{t}^{*}\left[l_{m}\right] & 1
\end{array}\right]\left[\begin{array}{c}
\mathbf{S}_{\mathbf{n}}[l] \\
\mathbf{S}_{\mathbf{n}}^{*}\left[l_{m}\right]
\end{array}\right] \\
& +\left[\begin{array}{c}
\mathbf{N}_{c}[l] \\
\mathbf{N}_{c}^{*}\left[l_{m}\right]
\end{array}\right] \\
& =\left[\begin{array}{cc}
\mathbf{B}[l] & 0 \\
0 & \mathbf{B}^{*}\left[l_{m}\right]
\end{array}\right] \\
& \times \underbrace{\left[\begin{array}{cc}
\left(1-\mathbf{Q}_{t}[l] \widetilde{\mathbf{Q}}_{t}^{*}\left[l_{m}\right]\right) & \left(\mathbf{Q}_{t}[l]-\widetilde{\mathbf{Q}}_{t}[l]\right) \\
\left(\mathbf{Q}_{t}^{*}\left[l_{m}\right]-\widetilde{\mathbf{Q}}_{t}^{*}\left[l_{m}\right]\right) & \left(1-\mathbf{Q}_{t}^{*}\left[l_{m}\right] \widetilde{\mathbf{Q}}_{t}[l]\right)
\end{array}\right]}_{\mathbf{Q}_{t t_{\text {tot }}}[l]} \\
& \times\left[\begin{array}{c}
\mathbf{S}[l] \\
\mathbf{S}^{*}\left[l_{m}\right]
\end{array}\right]+\left[\begin{array}{c}
\mathbf{N}_{c}[l] \\
\mathbf{N}_{c}^{*}\left[l_{m}\right]
\end{array}\right] .
\end{aligned}
$$

Under ideal conditions $\left(\widetilde{\mathbf{Q}}_{t}[l]=\mathbf{Q}_{t}[l]\right)$, the matrix $\mathbf{Q}_{t 1_{\text {tot }}}[l]$ is diagonalized and the remaining factors $\left(1-\mathbf{Q}_{t}[l] \widetilde{\mathbf{Q}}_{t}^{*}\left[l_{m}\right]\right)$ can be merged with $\mathbf{B}[l]$. The received symbol $\mathbf{Z}_{\text {tot }}[l]$ is then considered to be free of any transmitter IQ imbalance. As the predistortion is applied before the noise is added to the symbol, the transmitter IQ imbalance compensation is free from any noise enhancement.

We can now track the variation in channel based on

$$
\widetilde{\mathbf{B}}[l]=\frac{\mathbf{Z}[l]}{\left(1-\widetilde{\mathbf{Q}}_{t}[l] \widetilde{\mathbf{Q}}_{t}^{*}\left[l_{m}\right]\right) \mathbf{S}[l]} .
$$

The estimate of OFDM symbols is then obtained as

$$
\widetilde{\mathbf{S}}_{\text {tot }}[l]=\widetilde{\mathbf{B}}_{r_{-} \text {tot }}[l] \mathbf{B}_{\text {tot }}[l] \mathbf{Q}_{t_{\text {tot }}}[l] \widetilde{\mathbf{Q}}_{t_{\text {inv-tot }}}[l] \mathbf{S}_{\text {tot }}[l]
$$

where $\widetilde{\mathbf{Q}}_{t_{\text {inv_tot }}[l]}=\left[\begin{array}{cc}1 & -\widetilde{\mathbf{Q}}_{t}[l] \\ -\widetilde{\mathbf{Q}}_{t}^{*}\left[l_{m}\right] & 1\end{array}\right]$ and $\widetilde{\mathbf{B}}_{r_{-} \text {tot }}[l]=$ $\left[\begin{array}{cc}1 / \widetilde{\mathbf{B}}_{r}[l] & 0 \\ 0 & 1 / \widetilde{\mathbf{B}}_{r}^{*}\left[l_{m}\right]\end{array}\right]$. Here the term $\widetilde{\mathbf{B}}_{r}[l]=\widetilde{\mathbf{B}}[l](1-$ $\left.\widetilde{\mathbf{Q}}_{t}[l] \widetilde{\mathbf{Q}}_{t}^{*}\left[l_{m}\right]\right)$. A D-FEQ scheme based on predistortion transmitter IQ imbalance compensation is shown in Figure 2.

It should be noted that we can also apply a standard one-tap FEQ coefficient $\mathbf{W}_{a}[l]$ at the receiver for the direct estimation of the transmitted symbol, assuming transmitter IQ imbalance has been properly compensated by predistortion at the transmitter. The estimated symbol is then given as: $\widetilde{\mathbf{S}}[l]=\mathbf{W}_{a}[l] \mathbf{Z}[l]$. This one-tap FEQ is a reduced form compared to the two-tap FEQ used in (15). We now need only one training symbol for the estimation of the FEQ coefficient $\mathbf{W}_{a}[l]$. The FEQ coefficient can be initialized by LS or an adaptive RLS algorithm based on MMSE criterion.

3.3. Decoupled Transmitter/Receiver IQ Imbalance and Channel Distortion Compensation. The D-FEQ scheme can also be extended for the more general case with both transmitter and receiver IQ imbalance. In this case, the $\mathbf{D}_{\text {tot }}[l]$ can be decoupled as follows:

$$
\begin{aligned}
\mathbf{D}_{\text {tot }} & {[l] } \\
& =\left[\begin{array}{cc}
\mathbf{D}_{a}[l] & \mathbf{D}_{b}[l] \\
\mathbf{D}_{b}^{*}\left[l_{m}\right] & \mathbf{D}_{a}^{*}\left[l_{m}\right]
\end{array}\right] \\
= & \underbrace{\left[\begin{array}{cc}
1 & \mathbf{Q}_{r}[l] \\
\mathbf{Q}_{r}^{*}\left[l_{m}\right] & 1
\end{array}\right]}_{\mathbf{Q}_{\text {tot }}[l]} \underbrace{\left[\begin{array}{cc}
\mathbf{B}[l] & 0 \\
0 & \mathbf{B}^{*}\left[l_{m}\right]
\end{array}\right]}_{\mathbf{B}_{\text {tot }}[l]} \underbrace{\left[\begin{array}{cc}
1 & \mathbf{Q}_{t}[l] \\
\mathbf{Q}_{t}^{*}\left[l_{m}\right] & 1
\end{array}\right]}_{\mathbf{Q}_{\text {tot }}[l]}
\end{aligned}
$$

where $\mathbf{B}[l]=\mathbf{G}_{r a}[l] \mathbf{G}_{t a}[l] \mathbf{C}[l]$ is the composite channel, $\mathbf{Q}_{t}[l]=\mathbf{G}_{t b}[l] / \mathbf{G}_{t a}[l]$ is the transmitter IQ imbalance gain parameter, and $\mathbf{Q}_{r}[l]=\mathbf{G}_{r b}[l] / \mathbf{G}_{r a}^{*}\left[l_{m}\right]$ is the receiver IQ imbalance gain parameter. The $\mathbf{D}_{\text {tot }}[l]$ coefficients $\mathbf{D}_{a}[l]$ and $\mathbf{D}_{b}[l]$ can then be rewritten as

$$
\begin{aligned}
& \mathbf{D}_{a}[l]=\mathbf{B}[l]+\mathbf{Q}_{r}[l] \mathbf{Q}_{t}^{*}\left[l_{m}\right] \mathbf{B}^{*}\left[l_{m}\right], \\
& \mathbf{D}_{b}[l]=\mathbf{Q}_{t}[l] \mathbf{B}[l]+\mathbf{Q}_{r}[l] \mathbf{B}^{*}\left[l_{m}\right] .
\end{aligned}
$$

In the presence of both the transmitter and receiver IQ imbalance, it is not possible to obtain $\widetilde{\mathbf{Q}}_{t}[l], \widetilde{\mathbf{Q}}_{r}[l]$ and $\widetilde{\mathbf{B}}[l]$ estimates directly from the $\widetilde{\mathbf{D}}_{\text {tot- }}[l]$ matrix $(14)$. In order to obtain these estimates we first make an approximation, namely, that the second-order term $\mathbf{Q}_{r}[l] \mathbf{Q}_{t}^{*}\left[l_{m}\right]=0$ in $\mathbf{D}_{a}[l]$. This approximation is based on the fact that $\mathbf{G}_{t a}[l] \gg$ $\mathbf{G}_{t b}[l]$ and $\mathbf{G}_{r a}^{*}\left[l_{m}\right] \gg \mathbf{G}_{r b}[l]$ in practice. We can then estimate the channel $\widetilde{\mathbf{B}}[l] \simeq \widetilde{\mathbf{D}}_{a}[l]$ which is in line with (18). Equation (26) can now be written for $\widetilde{\mathbf{D}}_{b}[l]$ as follows:

$$
\widetilde{\mathbf{D}}_{b}[l]=\widetilde{\mathbf{Q}}_{t}[l] \widetilde{\mathbf{D}}_{a}[l]+\widetilde{\mathbf{Q}}_{r}[l] \widetilde{\mathbf{D}}_{a}^{*}\left[l_{m}\right]
$$



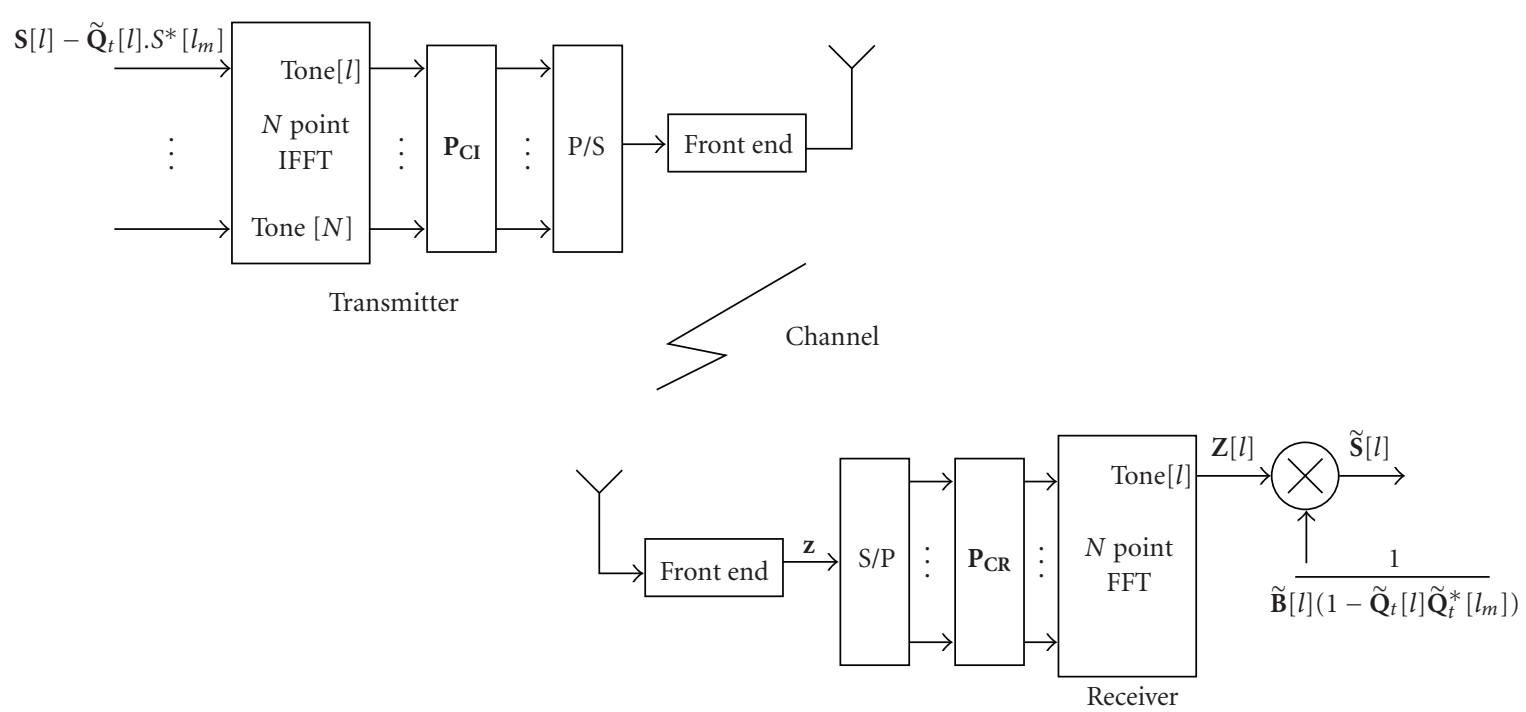

FIgURE 2: D-FEQ compensation scheme for transmitter IQ imbalance and channel distortion compensation. The system uses a predistortion-based compensation scheme for transmitter IQ imbalance. The channel distortion is compensated at the receiver.

In the case of FI transmitter and receiver IQ imbalance, the estimates can be straightforwardly obtained from (27) as

$$
\left[\begin{array}{c}
\widetilde{Q}_{t} \\
\widetilde{Q}_{r}
\end{array}\right]=\left[\begin{array}{cc}
\widetilde{\mathbf{D}}_{a}[2] & \widetilde{\mathbf{D}}_{a}^{*}[N] \\
\vdots & \vdots \\
\widetilde{\mathbf{D}}_{a}[l] & \widetilde{\mathbf{D}}_{a}^{*}\left[l_{m}\right] \\
\vdots & \vdots \\
\widetilde{\mathbf{D}}_{a}[N] & \widetilde{\mathbf{D}}_{a}^{*}[2]
\end{array}\right]^{\dagger}\left[\begin{array}{c}
\widetilde{\mathbf{D}}_{b}[2] \\
\vdots \\
\widetilde{\mathbf{D}}_{b}[l] \\
\vdots \\
\widetilde{\mathbf{D}}_{b}[N]
\end{array}\right] .
$$

In the case of FS transmitter and receiver IQ imbalance, the estimation of the gain parameters is to be performed for each tone individually. In order to obtain these estimates, we need at least two independent realizations of the channel, that is, $\mathbf{B}^{(1)}[l]$ and $\mathbf{B}^{(2)}[l]$, and hence $\widetilde{\mathbf{D}}_{a}^{(1)}[l], \widetilde{\mathbf{D}}_{a}^{(2)}[l]$ and $\widetilde{\mathbf{D}}_{b}^{(1)}[l] \widetilde{\mathbf{D}}_{b}^{(2)}[l]$, respectively. The estimates $\widetilde{\mathbf{Q}}_{t}[l]$ and $\widetilde{\mathbf{Q}}_{r}[l]$ can then be obtained from (27) as

$$
\left[\begin{array}{l}
\widetilde{\mathbf{Q}}_{t}[l] \\
\widetilde{\mathbf{Q}}_{r}[l]
\end{array}\right]=\left[\begin{array}{ll}
\widetilde{\mathbf{D}}_{a}^{(1)}[l] & \widetilde{\mathbf{D}}_{a}^{*(1)}\left[l_{m}\right] \\
\widetilde{\mathbf{D}}_{a}^{(2)}[l] & \widetilde{\mathbf{D}}_{a}^{*(2)}\left[l_{m}\right]
\end{array}\right]^{-1}\left[\begin{array}{l}
\widetilde{\mathbf{D}}_{b}^{(1)}[l] \\
\widetilde{\mathbf{D}}_{b}^{(2)}[l]
\end{array}\right] .
$$

For guaranteed invertibility of the matrix in (29) we should have $\widetilde{\mathbf{D}}_{a}^{(2)}[l] \neq \widetilde{\mathbf{D}}_{a}^{(1)}[l]$ and/or $\widetilde{\mathbf{D}}_{a}^{*(2)}\left[l_{m}\right] \neq \widetilde{\mathbf{D}}_{a}^{*(1)}\left[l_{m}\right]$.

It should be noted that the multipath diversity of the channel $\mathbf{B}[l]$, and hence $\widetilde{\mathbf{D}}_{a}[l]$, allows us to estimate transmitter/receiver IQ imbalance gain parameters in (28) and (29), respectively. The matrix should be well conditioned to obtain reliable estimates of IQ imbalance gain parameters. In general, we consider the coherence bandwidth of the channel to be small enough (or channel dispersion to be long enough) so that the channel response on the desired tone and its mirror tone are linearly independent. If the channel does not vary for a desired tone and its mirror tone over two independent channel realizations in (29), then a joint compensation scheme should be performed on that tone pair as in (15). On the other hand, (28) involves an overdetermined system of equation, thus we require only two pairs of $\widetilde{\mathbf{D}}_{a}[l]$ and $\widetilde{\mathbf{D}}_{a}\left[l_{m}\right]$ to be linearly independent for the matrix to be well conditioned, otherwise a joint compensation scheme should be performed for the entire OFDM symbol as in (15).

Equation (29) provides good estimates as long as $\mathbf{Q}_{r}[l] \mathbf{Q}_{t}^{*}\left[l_{m}\right] \simeq 0$, that is, both the transmitter and receiver IQ imbalance gain parameters are relatively small. The results are optimal if $\mathbf{Q}_{r}[l]=0$ (i.e., no receiver IQ imbalance; see Section 3.2) or $\mathbf{Q}_{t}[l]=0$ (i.e., no transmitter IQ imbalance). However, for large transmitter and receiver IQ imbalance values, the estimates obtained from (29) may not be accurate enough, resulting in only a partial compensation of the transmitter and receiver IQ imbalance. The same holds true for the estimates of the FI transmitter and receiver IQ imbalance gain parameters obtained from (28). From now on we will not further consider the FI case as the description of the FS case will also apply to the FI case.

If we compensate for the $\mathbf{D}_{\text {tot }}[l]$ matrix (removing the superscripts corresponding to different channel realizations), with the raw estimates of receiver IQ imbalance gain parameter, the resulting matrix $\mathbf{D}_{1_{\text {tot }}}[l]$ is given as

$$
\begin{aligned}
& \underbrace{\left[\begin{array}{cc}
1 & -\widetilde{\mathbf{Q}}_{r}[l] \\
-\widetilde{\mathbf{Q}}_{r}^{*}\left[l_{m}\right] & 1
\end{array}\right] \mathbf{D}_{\mathrm{tot}}[l]}_{\mathbf{D}_{\text {tot }}[l]} \\
& =\left[\begin{array}{cc}
1-\widetilde{\mathbf{Q}}_{r}[l] \mathbf{Q}_{r}^{*}\left[l_{m}\right] & \mathbf{Q}_{r}[l]-\widetilde{\mathbf{Q}}_{r}[l] \\
\mathbf{Q}_{r}^{*}\left[l_{m}\right]-\widetilde{\mathbf{Q}}_{r}^{*}\left[l_{m}\right] & 1-\widetilde{\mathbf{Q}}_{r}^{*}\left[l_{m}\right] \mathbf{Q}_{r}[l]
\end{array}\right] \\
& \quad \times\left[\begin{array}{cc}
\mathbf{B}[l] & 0 \\
0 & \mathbf{B}^{*}\left[l_{m}\right]
\end{array}\right]\left[\begin{array}{cc}
1 & \mathbf{Q}_{t}[l] \\
\mathbf{Q}_{t}^{*}\left[l_{m}\right] & 1
\end{array}\right] .
\end{aligned}
$$




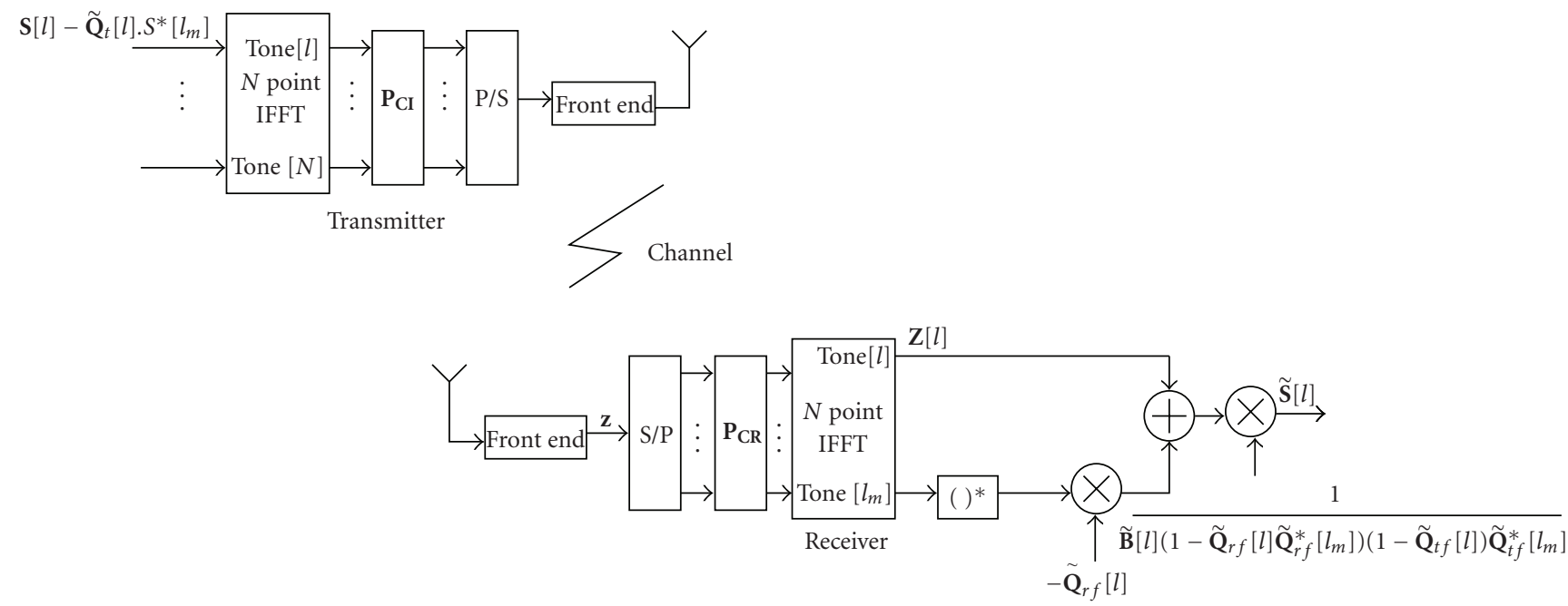

FIgURE 3: D-FEQ compensation scheme for transmitter and receiver IQ imbalance and channel distortion compensation. The system uses a predistortion-based compensation scheme for transmitter IQ imbalance. Both receiver IQ imbalance and the channel distortion are compensated at the receiver.

Equation (30) can be rewritten as:

$$
\begin{aligned}
& \mathbf{D}_{1_{\text {tot }}}[l] \\
& =\left[\begin{array}{cc}
\mathbf{D}_{a 1}[l] & \mathbf{D}_{b 1}[l] \\
\mathbf{D}_{b 1}^{*}\left[l_{m}\right] & \mathbf{D}_{a 1}^{*}\left[l_{m}\right]
\end{array}\right] \\
& =\underbrace{\left[\begin{array}{cc}
1 & \mathbf{Q}_{r 1}[l] \\
\mathbf{Q}_{r 1}^{*}\left[l_{m}\right] & 1
\end{array}\right]}_{\mathbf{Q}_{r 1_{\text {tot }}}[l]} \underbrace{\left[\begin{array}{cc}
\mathbf{B}_{1}[l] & 0 \\
0 & \mathbf{B}_{1}^{*}\left[l_{m}\right]
\end{array}\right]}_{\mathbf{B}_{1_{\text {tot }}}[l]} \underbrace{\left[\begin{array}{cc}
1 & \mathbf{Q}_{t 1}[l] \\
\mathbf{Q}_{t 1}^{*}\left[l_{m}\right] & 1
\end{array}\right]}_{\mathbf{Q}_{t 1_{\text {tot }}}[l]}
\end{aligned}
$$

which is similar to (25), and where $\mathbf{B}_{1}[l]=\mathbf{B}[l](1-$ $\left.\widetilde{\mathbf{Q}}_{r}[l] \mathbf{Q}_{r}^{*}\left[l_{m}\right]\right), \mathbf{Q}_{t 1}[l]=\mathbf{Q}_{t}[l], \mathbf{Q}_{r 1}[l]=\left(\mathbf{Q}_{r}[l]-\widetilde{\mathbf{Q}}_{r}[l]\right) /(1-$ $\left.\widetilde{\mathbf{Q}}_{r}^{*}\left[l_{m}\right] \mathbf{Q}_{r}[l]\right)$, and $\mathbf{Q}_{r 1}[l] \ll \mathbf{Q}_{r}[l]$. The $\mathbf{D}_{1_{\text {tot }}}[l]$ coefficients $\left(\mathbf{D}_{a 1}[l]\right.$ and $\left.\mathbf{D}_{b 1}[l]\right)$ are now written as

$$
\begin{aligned}
& \mathbf{D}_{a 1}[l]=\mathbf{B}_{1}[l]+\mathbf{Q}_{r 1}[l] \mathbf{Q}_{t 1}^{*}\left[l_{m}\right] \mathbf{B}_{1}^{*}\left[l_{m}\right], \\
& \mathbf{D}_{b 1}[l]=\mathbf{Q}_{t 1}[l] \mathbf{B}_{1}[l]+\mathbf{Q}_{r 1}[l] \mathbf{B}_{1}^{*}\left[l_{m}\right]
\end{aligned}
$$

which is similar to (26). Now the estimates $\tilde{\mathbf{D}}_{a 1}[l]$ and $\tilde{\mathbf{D}}_{b 1}[l]$ of $\mathbf{D}_{a 1}[l]$ and $\mathbf{D}_{b 1}[l]$, can be directly obtained from (30), with $\mathbf{D}_{\text {tot }}[l]$ replaced by the estimate $\tilde{\mathbf{D}}_{\text {tot }}[l]$, as follows:

$$
\left[\begin{array}{cc}
\tilde{\mathbf{D}}_{a 1}[l] & \tilde{\mathbf{D}}_{b 1}[l] \\
\tilde{\mathbf{D}}_{b 1}^{*}\left[l_{m}\right] & \tilde{\mathbf{D}}_{a 1}^{*}\left[l_{m}\right]
\end{array}\right]=\underbrace{\left[\begin{array}{cc}
1 & -\widetilde{\mathbf{Q}}_{r}[l] \\
-\widetilde{\mathbf{Q}}_{r}^{*}\left[l_{m}\right] & 1
\end{array}\right] \widetilde{\mathbf{D}}_{\text {tot }}[l]}_{\tilde{\mathbf{D}}_{1_{\text {tot }}}[l]} .
$$

Finally $\widetilde{\mathbf{Q}}_{r 1}[l]$ and an improved estimate $\widetilde{\mathbf{Q}}_{t 1}[l]$ of $\widetilde{\mathbf{Q}}_{t}[l]$ are obtained based on an expression similar to (29), with $\widetilde{\mathbf{D}}_{a}^{(1)}[l], \widetilde{\mathbf{D}}_{a}^{(2)}[l]$ and $\widetilde{\mathbf{D}}_{b}^{(1)}[l], \widetilde{\mathbf{D}}_{b}^{(2)}[l]$ replaced by $\widetilde{\mathbf{D}}_{a 1}^{(1)}[l], \widetilde{\mathbf{D}}_{a 1}^{(2)}[l]$ and $\widetilde{\mathbf{D}}_{b 1}^{(1)}[l], \widetilde{\mathbf{D}}_{b 1}^{(2)}[l]$.

Equations (29)-(33) may be repeated a number of times until $\widetilde{\mathbf{Q}}_{r i}[l] \simeq 0$, which corresponds to $\widetilde{\mathbf{D}}_{a i}[l] \simeq \widetilde{\mathbf{B}}_{i}[l]$, where $i$ represents the iteration number. After performing a sufficient number of iterations, the fine estimate of receiver IQ imbalance $\widetilde{\mathbf{Q}}_{r f}[l]$ can be derived from $\widetilde{\mathbf{Q}}_{r i}[l]$ as

$$
\widetilde{\mathbf{Q}}_{r f}[l]=\frac{\mathbf{Q}_{r 1}[l]+\tilde{\mathbf{Q}}_{r}[l]}{1+\mathbf{Q}_{r 1}[l] \widetilde{\mathbf{Q}}_{r}^{*}\left[l_{m}\right]},
$$

where $\mathbf{Q}_{r 1}[l]=\left(\mathbf{Q}_{r 2}[l]+\widetilde{\mathbf{Q}}_{r 1}[l]\right) /\left(1+\mathbf{Q}_{r 2}[l] \widetilde{\mathbf{Q}}_{r 1}^{*}\left[l_{m}\right]\right)$ and so on. For example, in a two-step iterative process, for instance, $\mathbf{Q}_{r 2}[l]$ is considered to be zero and therefore $\mathbf{Q}_{r 1}[l]=\widetilde{\mathbf{Q}}_{r 1}[l]$ and $\widetilde{\mathbf{Q}}_{r f}[l]=\left(\widetilde{\mathbf{Q}}_{r 1}[l]+\widetilde{\mathbf{Q}}_{r}[l]\right) /\left(1+\widetilde{\mathbf{Q}}_{r 1}[l] \widetilde{\mathbf{Q}}_{r}^{*}\left[l_{m}\right]\right)$. The fine estimate of the transmitter IQ imbalance $\widetilde{\mathbf{Q}}_{t f}[l]$ is the estimate $\widetilde{\mathbf{Q}}_{t i}[l]$ obtained from the last iteration.

It should be noted that the estimation of transmitter and receiver IQ imbalance gain parameters involve the division operation per tone, since the frequency response of a certain tone can be very small due to deep channel fading, the estimated IQ imbalance gain parameters may then not be accurate if the quantization level is limited or for poor signalto-noise conditions. From the hardware implementation point of view, the proposed estimation method may require high quantization level to cope with the existence of tones with very small gains. However, in order to obtain the best possible estimates, we can consider the availability of sufficiently long training symbols in order to reliably estimate IQ imbalance gain parameters during the estimation stage. The main advantage of the decoupled scheme is that we need to estimate the gain parameters only once during the estimation stage. For a slowly varying indoor multipath channel this can be a valid assumption. Thus, once we have reliable estimates of IQ imbalance gain parameters, we can then compensate the channel based on any commonly available methods. A longer training sequence will provide improved estimates due to a better noise averaging and will allow for reliable estimates. However, for a very limited quantization level it may be preferable to perform joint compensation on the affected tone pairs as given in (15). 
(1) Make an approximation, consider the second-order term $\mathbf{Q}_{r}[l] \mathbf{Q}_{t}^{*}\left[l_{m}\right]=0$ in $\mathbf{D}_{a}[l]=\mathbf{B}[l]+\mathbf{Q}_{r}[l] \mathbf{Q}_{t}^{*}\left[l_{m}\right] \mathbf{B}^{*}\left[l_{m}\right]$.

(2) (i) In the case of FI transmitter and receiver IQ imbalance, the raw estimates $\widetilde{Q}_{r}$ and $\widetilde{Q}_{t}$ are directly derived from $\widetilde{\mathbf{D}}_{b}[l]=\widetilde{Q}_{t}[l] \tilde{\mathbf{D}}_{a}[l]+\widetilde{Q}_{r}[l] \tilde{\mathbf{D}}_{a}^{*}\left[l_{m}\right]$.

(ii) In the case of FS transmitter and receiver IQ imbalance, the raw estimates $\widetilde{\mathbf{Q}}_{r}[l]$ and $\widetilde{\mathbf{Q}}_{t}[l]$ are derived from at least two independent realizations $\widetilde{\mathbf{D}}_{a}^{(1)}[l], \widetilde{\mathbf{D}}_{a}^{(2)}[l]$ and $\widetilde{\mathbf{D}}_{b}^{(1)}[l], \widetilde{\mathbf{D}}_{b}^{(2)}[l]$ in the equation $\widetilde{\mathbf{D}}_{b}^{(p)}[l]=\widetilde{\mathbf{Q}}_{t}[l] \widetilde{\mathbf{D}}_{a}^{(p)}[l]+\widetilde{\mathbf{Q}}_{r}[l] \widetilde{\mathbf{D}}_{a}^{*(p)}\left[l_{m}\right]$, where $p$ denotes a different realization.

(3) Compensate $\widetilde{\mathbf{D}}_{\text {tot }}[l]$ with the raw estimate of receiver IQ imbalance parameter $\widetilde{\mathbf{Q}}_{r}[l]$ to obtain the matrix $\widetilde{\mathbf{D}}_{i \text { tot }}[l]$ with coefficients $\widetilde{\mathbf{D}}_{a i}[l]$ and $\widetilde{\mathbf{D}}_{b i}[l]$, where $i$ is the iteration number.

(4) Obtain $\widetilde{\mathbf{Q}}_{r i}[l]$ and $\widetilde{\mathbf{Q}}_{t i}[l]$ by substituting coefficients $\widetilde{\mathbf{D}}_{a i}[l]$ and $\widetilde{\mathbf{D}}_{b i}[l]$ in step 2.

(5) Repeat steps 2-4, until $\widetilde{\mathbf{Q}}_{r i}[l]=0$.

(6) Fine estimate of receiver IQ imbalance is given as

where $\mathbf{Q}_{r 1}[l]=\left(\mathbf{Q}_{r 2}[l]+\widetilde{\mathbf{Q}}_{r 1}[l]\right) /\left(1+\mathbf{Q}_{r 2}[l] \widetilde{\mathbf{Q}}_{r 1}^{*}\left[l_{m}\right]\right)$ and so on.

$$
\widetilde{\mathbf{Q}}_{r f}[l]=\frac{\mathbf{Q}_{r 1}[l]+\widetilde{\mathbf{Q}}_{r}[l]}{1+\mathbf{Q}_{r 1}[l] \widetilde{\mathbf{Q}}_{r}^{*}\left[l_{m}\right]},
$$

(7) Fine estimate of transmitter IQ imbalance $\widetilde{\mathbf{Q}}_{t f}[l]$ is the estimate $\widetilde{\mathbf{Q}}_{t i}[l]$ obtained from the last iteration.

(8) Obtain the channel estimate:

$$
\widetilde{\mathbf{B}}[l]=\frac{\widetilde{\mathbf{D}}_{a}[l]-\widetilde{\mathbf{Q}}_{t f}^{*}\left[l_{m}\right] \widetilde{\mathbf{D}}_{b}[l]}{\left(1-\widetilde{\mathbf{Q}}_{t f}^{*}\left[l_{m}\right] \widetilde{\mathbf{Q}}_{t f}[l]\right)}
$$

Algorithm 1: D-FEQ scheme for the estimation of transmitter and receiver IQ imbalance parameters.

From the hardware implementation point of view, a tradeoff between quantization limit and the length of training sequence may be needed. The exploration of this trade-off is out of scope of this work.

Finally, the channel estimate $\widetilde{\mathbf{B}}[l]$ is derived based on (26) as

$$
\widetilde{\mathbf{B}}[l]=\frac{\widetilde{\mathbf{D}}_{a}[l]-\widetilde{\mathbf{Q}}_{t f}^{*}\left[l_{m}\right] \widetilde{\mathbf{D}}_{b}[l]}{\left(1-\widetilde{\mathbf{Q}}_{t f}^{*}\left[l_{m}\right] \widetilde{\mathbf{Q}}_{t f}[l]\right)}
$$

A complete algorithm description is provided in Algorithm 1.

Note. (i) From now, if the channel distortion is timevarying, only one training symbol is needed to reestimate the composite channel which can then be tracked based on

$$
\widetilde{\mathbf{B}}[l]=\frac{\left(\mathbf{Z}[l]-\widetilde{\mathbf{Q}}_{r f}[l] \mathbf{Z}^{*}\left[l_{m}\right]\right)}{\left(1-\widetilde{\mathbf{Q}}_{r f}[l] \widetilde{\mathbf{Q}}_{r f}^{*}\left[l_{m}\right]\right)\left(\mathbf{S}[l]+\widetilde{\mathbf{Q}}_{t f}[l] \mathbf{S} *\left[l_{m}\right]\right)}
$$

Similar to (20), we can once again formulate $\widetilde{\mathbf{D}}_{\text {tot }}[l]$ from the new composite channel estimate $\widetilde{\mathbf{B}}[l]$, the transmitter IQ imbalance gain parameter $\widetilde{\mathbf{Q}}_{t f}[l]$, and the receiver IQ imbalance gain parameter $\tilde{\mathbf{Q}}_{r f}[l]$. A 2-tap FEQ is then employed for the estimation of the transmitted OFDM symbol $\tilde{\mathbf{S}}[l]$.

(ii) In the case of predistortion of transmitted symbols (Section 3.2), we can track the variation in channel as

$$
\widetilde{\mathbf{B}}[l]=\frac{\left(\mathbf{Z}[l]-\widetilde{\mathbf{Q}}_{r f}[l] \mathbf{Z}^{*}\left[l_{m}\right]\right)}{\left(1-\widetilde{\mathbf{Q}}_{r f}[l] \widetilde{\mathbf{Q}}_{r f}^{*}\left[l_{m}\right]\right)\left(1-\widetilde{\mathbf{Q}}_{t f}[l] \widetilde{\mathbf{Q}}_{t f}^{*}\left[l_{m}\right]\right) \mathbf{S}[l]} .
$$

The estimate of OFDM symbols is then obtained as

$$
\begin{aligned}
\widetilde{\mathbf{S}}_{\text {tot }}[l]= & \widetilde{\mathbf{B}}_{r_{\text {tot }}}[l] \widetilde{\mathbf{Q}}_{r_{\text {invtot }}}[l] \mathbf{Q}_{r_{\text {tot }}}[l] \\
& \times \mathbf{B}_{\text {tot }}[l] \mathbf{Q}_{t_{\text {tot }}}[l] \widetilde{\mathbf{Q}}_{t_{\text {invtot }}}[l] \mathbf{S}_{\text {tot }}[l],
\end{aligned}
$$

where $\tilde{\mathbf{Q}}_{t_{\text {inv_tot }}[l]}=\left[\begin{array}{cc}1 & -\widetilde{\mathbf{Q}}_{t f}[l] \\ -\widetilde{\mathbf{Q}}_{t f}^{*}\left[l_{m}\right] & 1\end{array}\right], \quad \widetilde{\mathbf{Q}}_{r_{\text {inv_tot }}[l]}=$ $\left[\begin{array}{cc}1 & -\widetilde{\mathbf{Q}}_{r f}[l] \\ -\widetilde{\mathbf{Q}}_{r f}^{*}\left[l_{m}\right] & 1\end{array}\right]$, and $\widetilde{\mathbf{B}}_{r_{-} \text {tot }}[l]=\left[\begin{array}{cc}1 / \widetilde{\mathbf{B}}_{r}[l] & 0 \\ 0 & 1 / \widetilde{\mathbf{B}}_{r}^{*}\left[l_{m}\right]\end{array}\right]$. Here the term $\widetilde{\mathbf{B}}_{r}[l]=\widetilde{\mathbf{B}}[l]\left(1-\widetilde{\mathbf{Q}}_{r f}[l] \widetilde{\mathbf{Q}}_{r f}^{*}\left[l_{m}\right]\right)\left(1-\widetilde{\mathbf{Q}}_{t f}[l] \widetilde{\mathbf{Q}}_{t f}^{*}\left[l_{m}\right]\right)$.

The D-FEQ scheme based on (38) for the compensation of transmitter and receiver IQ imbalance is shown in Figure 3. Similar to Section 3.2, we can also apply a standard one-tap FEQ coefficient $\mathbf{W}_{a}[l]$ after the compensation of receiver IQ imbalance in order to directly estimate the transmitted symbol. The FEQ coefficient can be initialized by only one training symbol by LS or an RLS adaptive algorithm.

Based on (38), we can now also derive the improvement in IRR after the compensation of only transmitter and receiver IQ imbalance, and without the compensation of channel distortion in the received signal. In this case the received signal $\mathbf{Z}_{\text {comp }}[l]$ is given as

$$
\begin{aligned}
& \mathbf{Z}_{\text {comp }}[l]
\end{aligned}
$$

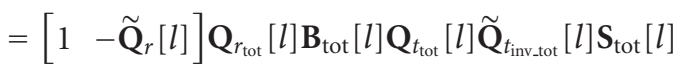

$$
\begin{aligned}
& =\left[\begin{array}{l}
\left(\mathbf{B}[l] \mathbf{Q}_{r_{\text {diff }}}[l] \mathbf{Q}_{t_{\text {diffl }}}[l]+\mathbf{Q}_{r_{\text {diff } 2}}[l] \mathbf{B}^{*}\left[l_{m}\right] \mathbf{Q}_{t_{\text {diff } 2}}^{*}\left[l_{m}\right]\right) \\
\left(\mathbf{B}[l] \mathbf{Q}_{r_{\text {diff }}}[l] \mathbf{Q}_{t_{\text {diff } 2}}[l]+\mathbf{Q}_{r_{\text {diff } 2}}[l] \mathbf{B}^{*}\left[l_{m}\right] \mathbf{Q}_{t_{\text {diff }}}^{*}\left[l_{m}\right]\right)
\end{array}\right]^{T} \\
& \times\left[\begin{array}{c}
\mathbf{S}[l] \\
\mathbf{S}^{*}\left[l_{m}\right]
\end{array}\right]
\end{aligned}
$$




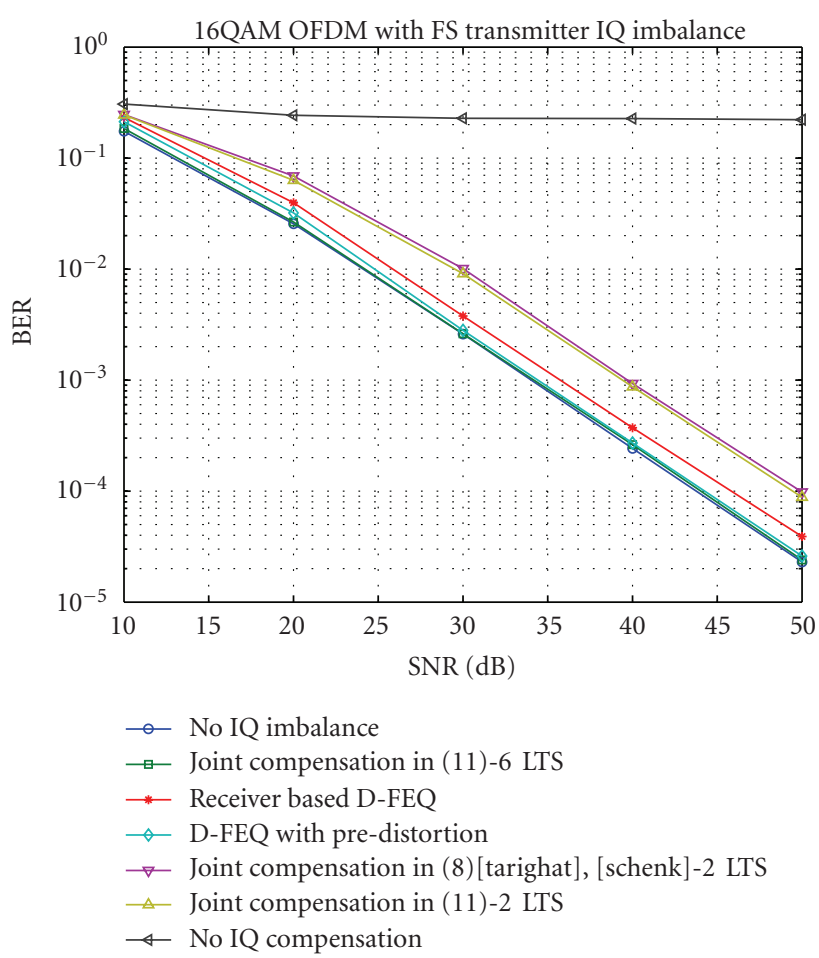

(a) BER versus SNR for transmitter IQ imbalance

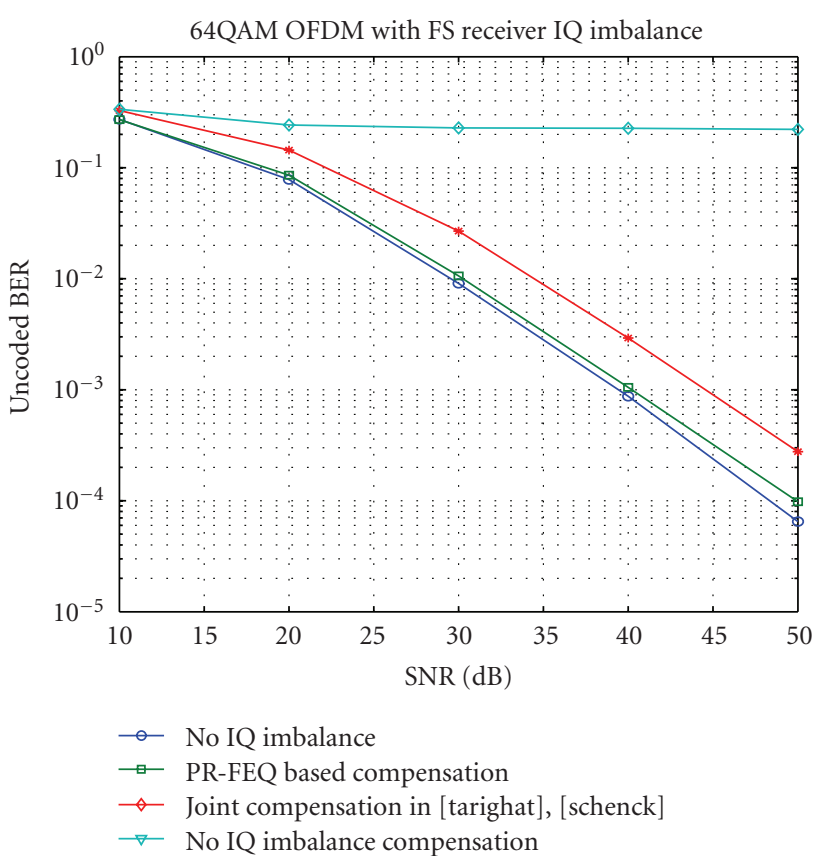

(b) BER versus SNR for receiver IQ imbalance

FIgURE 4: BER versus SNR for OFDM system. (a) D-FEQ based transmitter IQ imbalance compensation for a 16QAM OFDM system. Frequency independent amplitude imbalance of $g_{t}, g_{r}=5 \%$ and phase imbalance of $\phi_{t}, \phi_{r}=5^{\circ}$. The front-end filter impulse responses are $\mathbf{h}_{t i}=\mathbf{h}_{r i}=\left[\begin{array}{lll}0.01, & 0.5 & 0.06\end{array}\right]$ and $\mathbf{h}_{t q}=\mathbf{h}_{r q}=\left[\begin{array}{lll}0.06 & 0.5, & 0.01\end{array}\right]$. (b) PR-FEQ-based receiver IQ imbalance compensation for a 64QAM OFDM system. Frequency independent amplitude imbalance of $g_{t}, g_{r}=10 \%$ and phase imbalance of $\phi_{t}, \phi_{r}=10^{\circ}$. The front-end filter impulse responses are $\mathbf{h}_{t i}=\mathbf{h}_{r i}=\left[\begin{array}{lll}0.01, & 0.5 & 0.06\end{array}\right]$ and $\mathbf{h}_{t q}=\mathbf{h}_{r q}=\left[\begin{array}{lll}0.06 & 0.5, & 0.01\end{array}\right]$.

where $\mathbf{Q}_{t_{\text {diff }}}[l]=\left(1-\mathbf{Q}_{t}[l] \widetilde{\mathbf{Q}}_{t f}^{*}\left[l_{m}\right]\right), \mathbf{Q}_{t_{\text {diff }}}[l]=\left(\mathbf{Q}_{t}[l]-\right.$ $\left.\widetilde{\mathbf{Q}}_{t f}[l]\right), \mathbf{Q}_{r_{\text {diff }}}[l]=\left(1-\widetilde{\mathbf{Q}}_{r f}[l] \mathbf{Q}_{r}^{*}\left[l_{m}\right]\right)$, and $\mathbf{Q}_{r_{\text {diff }}}[l]=$ $\left(\mathbf{Q}_{r}[l]-\widetilde{\mathbf{Q}}_{r f}[l]\right)$.

The IRR improvement is obtained as

$$
\begin{aligned}
& \mathbf{I R R}_{\text {comp }}[l] \\
& =10 \log _{10} \\
& \quad \times\left(\frac{\left|\mathbf{B}[l] \mathbf{Q}_{r_{\text {diff }}}[l] \mathbf{Q}_{t_{\text {diffl }}}[l]+\mathbf{Q}_{r_{\text {diff }}}[l] \mathbf{B}^{*}\left[l_{m}\right] \mathbf{Q}_{t_{\text {diff }}}^{*}\left[l_{m}\right]\right|^{2}}{\left|\mathbf{B}[l] \mathbf{Q}_{r_{\text {diff }}}[l] \mathbf{Q}_{t_{\text {diff }}}[l]+\mathbf{Q}_{r_{\text {diff }}}[l] \mathbf{B}^{*}\left[l_{m}\right] \mathbf{Q}_{t_{\text {diff }}}^{*}\left[l_{m}\right]\right|^{2}}\right)
\end{aligned}
$$

The improvement in $\mathbf{I R R}_{\text {comp }}[l]$ performance when compared to IRR $[l]$ in (10) is later illustrated in Section 4.

\subsection{Decoupled Receiver IQ Imbalance and Channel Distortion} Compensation. In the case of only receiver IQ imbalance and no transmitter IQ imbalance $\left(\mathbf{G}_{t a}[l]=1, \mathbf{G}_{t b}[l]=\right.$ 0 ), a reduced form of the D-FEQ estimation/compensation scheme in Section 3.3 can be used. In this case, the receiver IQ imbalance gain parameter $\mathbf{Q}_{r}[l]=\mathbf{G}_{r b}[l] / \mathbf{G}_{r a}^{*}\left[l_{m}\right]$ and the composite channel $\mathbf{B}[l]=\mathbf{G}_{r a}[l] \mathbf{C}[l]$ can be directly derived from the $\mathbf{D}_{\text {tot- }}[l]$ coefficients. The estimates $\widetilde{\mathbf{Q}}_{r}[l]$ and $\widetilde{\mathbf{B}}[l]$ of $\mathbf{Q}_{r}[l]$ and $\mathbf{B}[l]$ are given as

$$
\begin{aligned}
\widetilde{\mathbf{Q}}_{r}[l] & =\frac{\widetilde{\mathbf{D}_{b}}[l]}{\widetilde{\mathbf{D}_{a}^{*}}\left[l_{m}\right]}, \\
\widetilde{\mathbf{B}}[l] & =\widetilde{\mathbf{D}}_{a}[l] .
\end{aligned}
$$

The D-FEQ scheme first estimates $\mathbf{D}_{\text {tot- }}[l]$ based on (13), and then derives $\widetilde{\mathbf{Q}}_{r}[l]$ from the $\widetilde{\mathbf{D}}_{\text {tot- }}[l]$ coefficients based on (41). This implies that to estimate the receiver IQ imbalance gain parameter $\mathbf{Q}_{r}[l]$, first $\mathbf{D}_{a}[l], \mathbf{D}_{b}[l]$ and then $\mathbf{D}_{a}\left[l_{m}\right], \mathbf{D}_{b}\left[l_{m}\right]$ have to be estimated. However, estimating the latter coefficient $\mathbf{D}_{b}\left[l_{m}\right]$ may not be useful per se especially so when the mirror tones, for instance, consist of pilot tones. We therefore propose an alternative scheme where $\widetilde{\mathbf{Q}}_{r}[l]$ can be estimated directly from the training symbols, thus saving on the computational cost involved in the estimation of the $\mathbf{D}_{\text {tot- }}[l]$ coefficients.

We consider a specific sequence of $M_{l}$ so-called phaserotated LTS. All the training symbols are identical up to a different phase rotation $e^{j \Phi^{(i)}}$ where $i$ represents the training 
symbol number, that is, $\mathbf{S}^{(i)}=\mathbf{S} e^{j \Phi^{(i)}}$. The phase rotations $\Phi^{(i)}$ can be between $0 \cdots 2 \pi$ radians. At the receiver side, we multiply the complex conjugate of the mirror symbol $\mathbf{Z}_{m}^{*(i)}[l]$ with a factor $\mathbf{V}_{b}[l]$ (to be defined) and add the output of this product to the received symbol $\mathbf{Z}^{(i)}[l]$, this results in

$$
\begin{aligned}
& \mathbf{Z}_{q}^{(i)}[l] \\
& =\left[\begin{array}{ll}
1 & \mathbf{V}_{b}[l]
\end{array}\right]\left[\begin{array}{c}
\mathbf{Z}^{(i)}[l] \\
\mathbf{Z}^{*(i)}\left[l_{m}\right]
\end{array}\right] \\
& =\left[\begin{array}{ll}
1 & \mathbf{V}_{b}[l]
\end{array}\right] \\
& \times\left(\left[\begin{array}{cc}
1 & \mathbf{Q}_{r}[l] \\
\mathbf{Q}_{r}^{*}\left[l_{m}\right] & 1
\end{array}\right]\left[\begin{array}{c}
e^{j \Phi^{(i)}} \mathbf{B}[l] \mathbf{S}[l] \\
e^{-j \Phi^{(i)}} \mathbf{B}^{*}\left[l_{m}\right] \mathbf{S}^{*}\left[l_{m}\right]
\end{array}\right]\right. \\
& \left.+\left[\begin{array}{cc}
\mathbf{G}_{r a}[l] & \mathbf{G}_{r b}[l] \\
\mathbf{G}_{r b}^{*}\left[l_{m}\right] & \mathbf{G}_{r a}^{*}\left[l_{m}\right]
\end{array}\right]\left[\begin{array}{c}
\mathbf{N}^{(i)}[l] \\
\mathbf{N}^{*(i)}\left[l_{m}\right]
\end{array}\right]\right) .
\end{aligned}
$$

If $\mathbf{V}_{b}[l]=-\mathbf{Q}_{r}[l]=-\mathbf{G}_{r b}[l] / \mathbf{G}_{r a}^{*}\left[l_{m}\right]$, then the contribution from $\mathbf{S}^{*}\left[l_{m}\right]$ and $\mathbf{N}^{*(i)}\left[l_{m}\right]$ is eliminated, and so the symbol $\mathbf{Z}_{q}^{(i)}[l]$ can be considered to be free of receiver IQ imbalance. Finally (42) can be re-written as

$$
\mathbf{Z}_{q}^{(i)}[l]=\mathbf{Q}_{x}[l] e^{j \Phi^{(i)}} \mathbf{B}[l] \mathbf{S}[l]+\mathbf{G}_{x}[l] \mathbf{N}^{(i)}[l]
$$

where the scaling term $\mathbf{Q}_{x}[l]=\left(1-\mathbf{Q}_{r}[l] \mathbf{Q}_{r}^{*}\left[l_{m}\right]\right)$ and $\mathbf{G}_{x}[l]=\left[\mathbf{G}_{r a}[l]-\left(\left(\mathbf{G}_{r b}[l] \cdot \mathbf{G}_{r b_{m}}^{*}\left[l_{m}\right]\right) / \mathbf{G}_{r a_{m}}^{*}\left[l_{m}\right]\right)\right]$ can be merged with the channel.

In the noiseless case, we can then relate pairs of received symbols as follows:

$$
\begin{gathered}
\mathbf{Z}_{q}^{(j)}[l]=e^{j \Omega} \mathbf{Z}_{q}^{(i)}[l], \\
\mathbf{Z}^{(j)}[l]-e^{j \Omega} \mathbf{Z}^{(i)}[l]=\left(e^{j \Omega} \mathbf{Z}^{*(i)}\left[l_{m}\right]-\mathbf{Z}^{*(j)}\left[l_{m}\right]\right) \mathbf{V}_{b}[l],
\end{gathered}
$$

where $\Omega=\Phi^{(j)}-\Phi^{(i)}, i=1 \cdots M_{l}-1, j=i+1 \cdots M_{l}$, and $j>i$. In matrix form, (44) can be written as

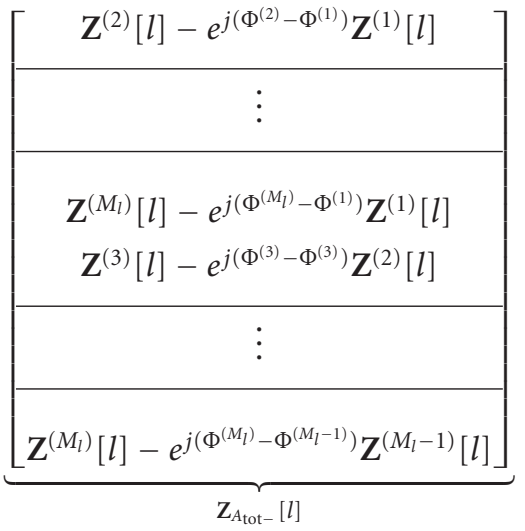

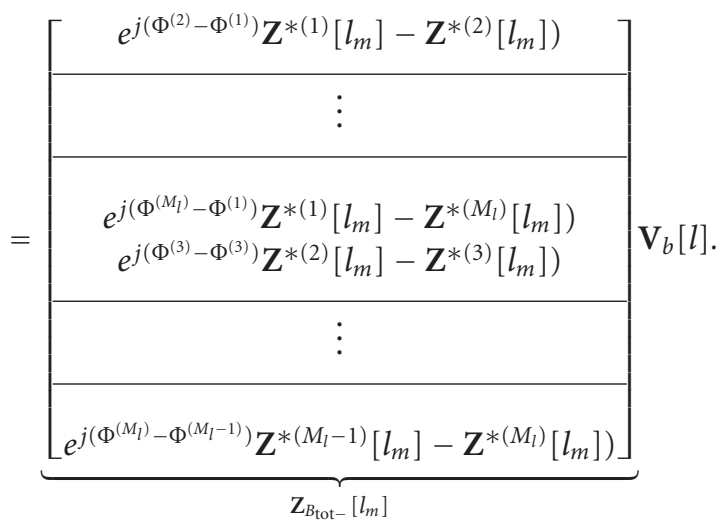

Finally the factor $\mathbf{V}_{b}[l]$ is obtained as

$$
\mathbf{V}_{b}[l]=\mathbf{Z}_{B_{\text {tot- }}}^{\dagger}\left[l_{m}\right] \mathbf{Z}_{A_{\text {tot- }}}[l] .
$$

The total number of valid pairs $(i, j)$ that can be considered in (45) is $N_{p}=C_{2}^{M_{l}}-N_{\Omega}$ where $C_{a}^{b}=b ! / a !(b-a) !$ and $N_{\Omega}$ is the total number of pairs with $\Omega=0, \pi$, and $2 \pi$ radians. We do not consider tone pairs with $\Omega=0, \pi, 2 \pi$ as these lead to ill-conditioning in (45). $N_{p}$ shows that as the number of training symbols is increased, we also have additional tone pairs that can be included in (45), leading to an improved estimation. The coefficient $\mathbf{V}_{b}[l]$ so obtained provides an estimate of the receiver IQ imbalance gain parameter, $\mathbf{V}_{b}[l]=\widetilde{\mathbf{Q}}_{r}[l]$, and is independent of the channel characteristic. Finally, in the case of FI receiver IQ imbalance, we can average the $\mathbf{V}_{b}[l]$ over all the tones to obtain an improved estimate $V_{b}=1 / N \sum_{l=1}^{N} \mathbf{V}_{b}[l]$. The composite channel is estimated after the compensation of the receiver IQ imbalance based on

$$
\widetilde{\mathbf{B}}[l]=\frac{\left(\mathbf{Z}[l]+\mathbf{V}_{b}[l] \mathbf{Z}^{*}\left[l_{m}\right]\right)}{\left(1-\mathbf{V}_{b}[l] \mathbf{V}_{b}^{*}\left[l_{m}\right]\right) \mathbf{S}[l]}
$$

Again, only one training symbol is needed to estimate the channel. Similar to (20), we can once again formulate $\widetilde{\mathbf{D}}_{\text {tot }}[l]$ from the new composite channel estimate $\widetilde{\mathbf{B}}[l]$, the receiver IQ imbalance gain parameter $\mathbf{V}_{b}[l]=\widetilde{\mathbf{Q}}_{r}[l]$, in order to estimate the transmitted OFDM symbol $\widetilde{\mathbf{S}}[l]$.

Alternatively, a one-tap FEQ coefficient $\mathbf{W}_{a}[l]$ can be applied for the direct estimation of transmitted symbol, given as

$$
\widetilde{\mathbf{S}}[l]=\mathbf{W}_{a}[l]\left[\begin{array}{ll}
1 & \mathbf{V}_{b}[l]
\end{array}\right]\left[\begin{array}{c}
\mathbf{Z}[l] \\
\mathbf{Z}\left[l_{m}\right]
\end{array}\right] .
$$

The FEQ coefficient is initialized by LS or an adaptive RLS training-based algorithm. Only one training symbol is needed to initialize $\mathbf{W}_{a}[l]$. We will refer to this phase-rotated LTS-based estimation scheme as PR-FEQ.

\section{Simulation}

We have simulated an OFDM system (similar to IEEE 802.11a) to evaluate the performance of the compensation 
schemes for transmitter and/or receiver IQ imbalance. The parameters used in the simulation are as follows: OFDM symbol length $N=64$ and cyclic prefix length $v=16$. We consider a quasistatic multipath channel of $L=4$ taps. The taps of the multipath channel are chosen independently with complex Gaussian distribution.

Figures 4(a) and 4(b) show the obtained bit error rate (BER) versus signal-to-noise ratio (SNR) performance curves. The BER performance results depicted are obtained by taking the average of the BER curves over $10^{4}$ independent channels. Figure 4(a) considers the presence of only transmitter IQ imbalance in a 16QAM OFDM system. The transmitter filter impulse responses are $\mathbf{h}_{t i}=\left[\begin{array}{lll}0.01, & 0.5 & 0.06\end{array}\right]$ and $\mathbf{h}_{t q}=\left[\begin{array}{lll}0.06 & 0.5, & 0.01\end{array}\right]$ and the transmitter frequency independent amplitude and phase imbalances are $g_{t}=5 \%$ and $\phi_{t}=5^{\circ}$, respectively. During the estimation phase of the transmitter IQ imbalance gain parameter, we consider $M_{l}=$ 6 LTS, while $M_{l}=2$ LTS are employed during the estimation phase of only the channel characteristics, as this is the minimal requirement for the joint estimation/compensation scheme in (12) [11, 17]. The figure shows the performance curves obtained for the proposed receiver-based D-FEQ compensation, predistortion-based D-FEQ compensation, and the joint compensation scheme (both ZF-based (12) [11, 17], and MMSE-based (15)), together with a system with no IQ imbalance and a system with no IQ imbalance compensation algorithm included. The figure also shows the performance curve obtained for joint compensation scheme (15) when $M_{l}=6$ LTS are employed. It can be seen that the proposed D-FEQ compensation schemes provide a very good performance. The results obtained with the predistortion-based D-FEQ scheme are very close to the case when there is no IQ imbalance in the system. The difference between the predistortion scheme and the joint compensation scheme (12) and (15) is almost $6 \mathrm{~dB}$ at BER of $10^{-3}$. For a large number of training symbols $\left(M_{l}=6\right.$ LTS), the MMSE-based joint compensation scheme provides the same performance as the case with no IQ imbalance. But for an extremely short training overhead $\left(M_{l}=2\right.$ LTS), the MMSE-based joint compensation scheme together with ZF-based joint compensation scheme give relatively poor performance compared to the D-FEQ scheme, this is mainly because of poor noise averaging. Thus the proposed $\mathrm{D}-\mathrm{FEQ}$ scheme is useful when the training overhead is limited.

Figure 4(b) considers the presence of only receiver IQ imbalance in a 64QAM OFDM system. The receiver filter impulse responses are $\mathbf{h}_{r i}=\left[\begin{array}{lll}0.01, & 0.5 & 0.06\end{array}\right]$ and $\mathbf{h}_{r q}=$ $\left[\begin{array}{ll}0.06 & 0.5,0.01\end{array}\right]$ and the receiver frequency independent amplitude and phase imbalances are $g_{r}=10 \%$ and $\phi_{r}=10^{\circ}$, respectively. Here we use the PR-FEQ scheme instead of the D-FEQ-based compensation scheme. During the estimation phase of the receiver IQ imbalance gain parameters, we consider $M_{l}=4$ identically phase-rotated LTS. The phase rotations of the symbols are $\Phi=0, \pi / 4, \pi / 2,3 \pi / 4$. Once again, we employ only $M_{l}=2$ LTS during the estimation phase of channel characteristics. The proposed scheme again provides an efficient compensation performance with a very small training overhead requirement.
Figures $5(a)-5(d)$ consider the presence of both the transmitter and receiver IQ imbalance in a 64QAM OFDM system. The transmitter and receiver filter impulse responses are $\mathbf{h}_{t i}=\mathbf{h}_{r i}=\left[\begin{array}{lll}0.01, & 0.5 & 0.06\end{array}\right]$, and $\mathbf{h}_{t q}=\mathbf{h}_{r q}=$ $\left[\begin{array}{lll}0.06 & 0.5,0.01\end{array}\right]$. In Figure 5(a), 5(b), and 5(d) the transmitter and receiver frequency independent amplitude and phase imbalances are $g_{t}=g_{r}=10 \%$ and $\phi_{t}=\phi_{r}=$ $20^{\circ}$, respectively. It should be noted that these imbalance levels may be higher than the level typically observed in a practical receiver. However, we consider such an extreme case to evaluate the robustness/effectiveness of the proposed compensation schemes. Here, we first consider $M_{l}=8$ LTS during the estimation phase of transmitter and receiver IQ imbalance gain parameters and then $M_{l}=2$ LTS during the estimation phase of only the channel characteristics.

Figures 5(a) and 5(b) illustrate the number of iterations required to perform adequate compensation for the given values of the IQ imbalance parameters. Both simulation results are obtained at $\mathrm{SNR}=40 \mathrm{~dB}$. Figure 5(a) shows the convergence of the transmitter and receiver IQ imbalance gain estimates to their ideal values. The curves measure the IQ imbalance gain estimates as the mean of the absolute values for all $N$ tones of an OFDM symbol (i.e., $\Xi\left\{\left|\widetilde{\mathbf{Q}}_{t}[l]\right|\right\}$ and $\Xi\left\{\left|\widetilde{\mathbf{Q}}_{r}[l]\right|\right\}$, where $\Xi$ is the expectation operator). It can be observed that 3-4 iterations can already provide sufficiently good estimates.

Figure 5(b) shows the image rejection ratio (IRR) observed for a system impaired with transmitter and receiver IQ imbalance. The figure shows that the IQ imbalance in our case is quite severe, in that with no compensation scheme in place the IRR is only $5-15 \mathrm{~dB}(10)$. The figure also shows the improvement in IRR in the presence of predistortion and a transmitter/receiver IQ imbalance compensation scheme (40). It can be observed that with only 1 iteration (Iter $=1$ ), an IRR improvement of around $30 \mathrm{~dB}$ is already obtained. Further improvement in IRR can be obtained by performing few more iterations. This improvement is however limited as the IRR saturates after a certain number of iterations due to the noise. In our simulations, we obtained a further improvement of around $10 \mathrm{~dB}$ after performing 3 more iterations $($ Iter $=4)$.

Figure 5(c) shows the mean IRR improvement with D-FEQ scheme for different values of transmitter/receiver frequency independent IQ imbalance. The mean IRR results are obtained over $10^{4}$ independent channels. The figure shows that D-FEQ scheme with predistortion provides a mean IRR of $44 \mathrm{~dB}$ at $40 \mathrm{~dB}$ SNR. This provides an IRR improvement of $3 \mathrm{~dB}$ even when extremely small amount of transmitter/receiver frequency independent IQ imbalance of $g_{t}=g_{r}=0.5 \%$ and $\phi_{t}=\phi_{r}=0.5^{\circ}$ is considered. The IRR improvement is significant when large transmitter and receiver IQ imbalance values are present. The figure shows that for extremely small amount of IQ imbalance $g_{t}=g_{r}=$ $0.1 \%$ and $\phi_{t}=\phi_{r}=0.1^{\circ}$ the IRR improvement with DFEQ scheme is similar to the system with no IQ imbalance compensation. Under these conditions, the deterioration in BER will be the same as the one obtained with D-FEQ scheme. But as the compensation performance obtained with 


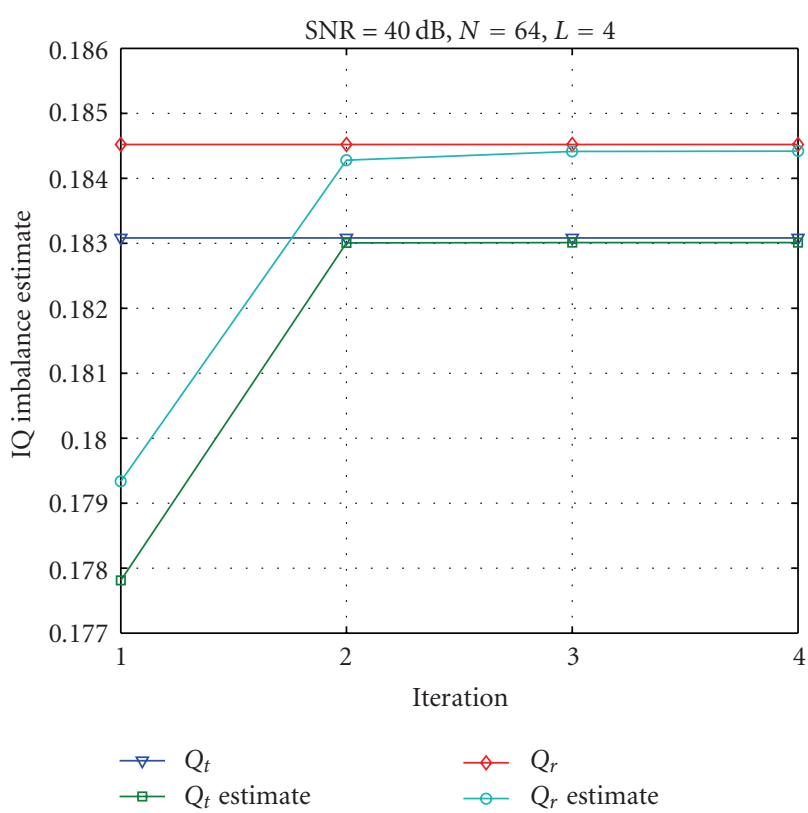

(a) IQ imbalance estimation with iterative method

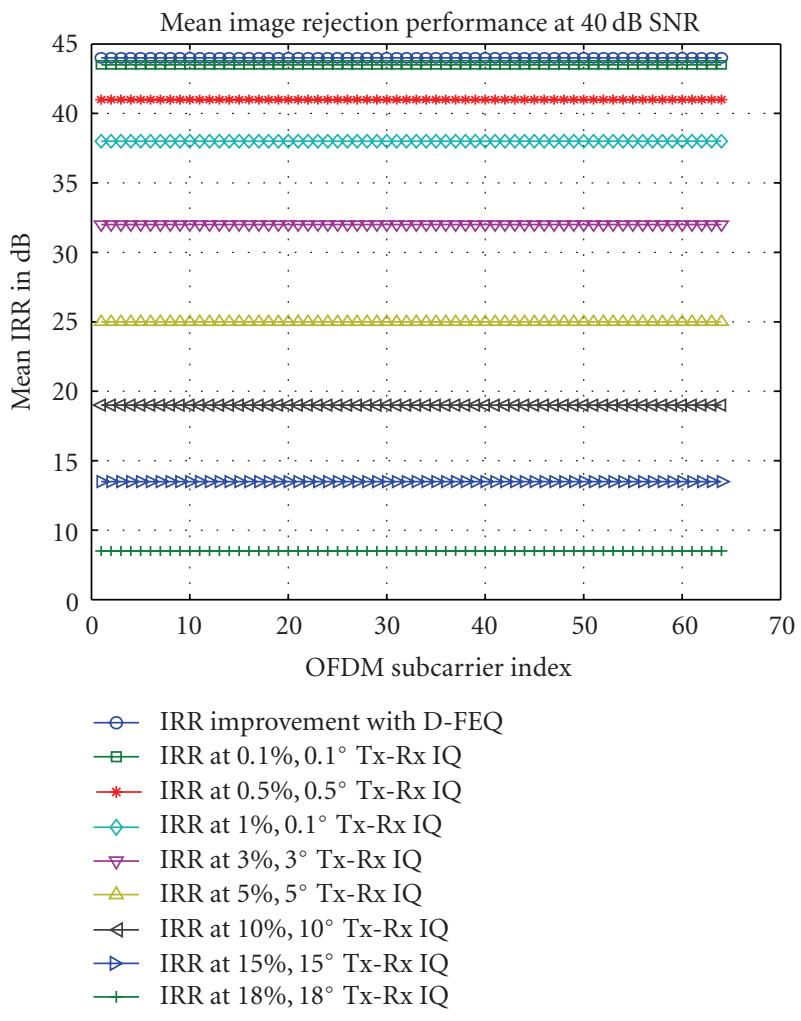

(c) Mean IRR performance

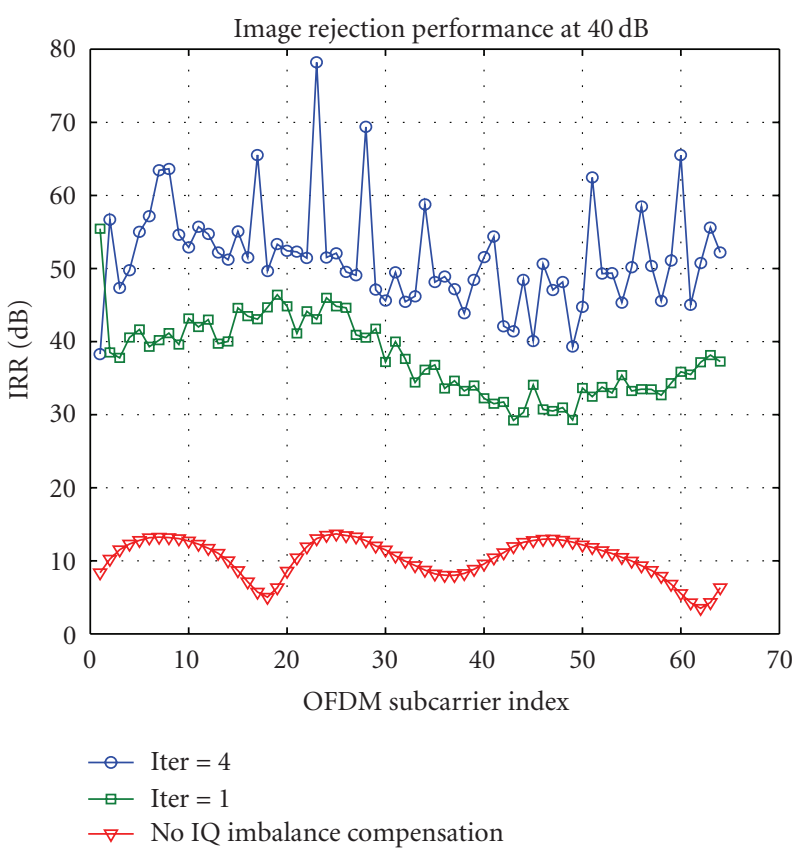

(b) IRR with transmitter/receiver IQ imbalance

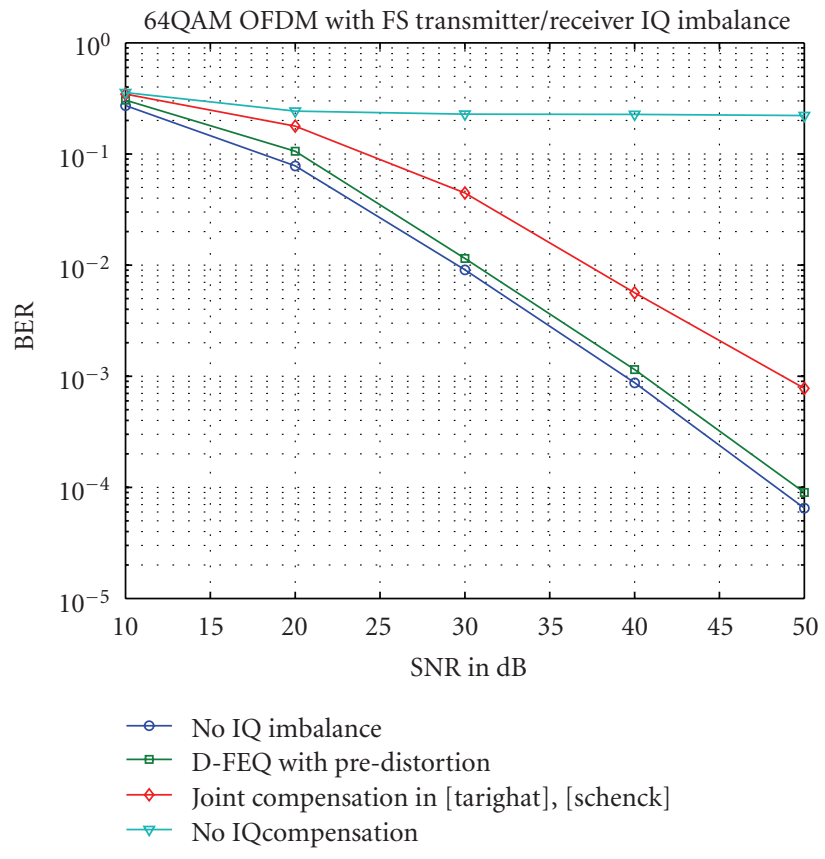

FIGURE 5: Performance results for 64QAM OFDM system with transmitter and receiver IQ imbalance. D-FEQ scheme with predistortion based compensation is implemented. (a)-(d) The front-end filter impulse responses are $\mathbf{h}_{t i}=\mathbf{h}_{r i}=\left[\begin{array}{lll}0.01,0.5 & 0.06\end{array}\right]$ and $\mathbf{h}_{t q}=\mathbf{h}_{r q}=$ $[0.060 .5,0.01]$. (a), (b), and (d) Performance results with frequency independent amplitude imbalance of $g_{t}, g_{r}=10 \%$ and phase imbalance of $\phi_{t}, \phi_{r}=20^{\circ}$. 


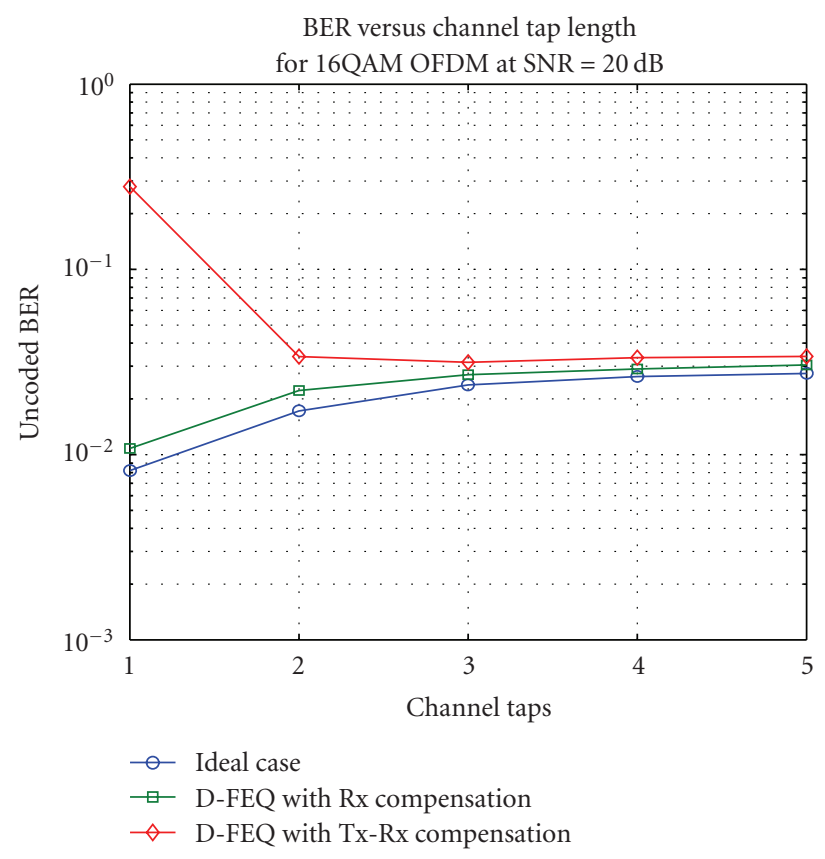

(a) BER versus channel tap length at $20 \mathrm{~dB}$ SNR

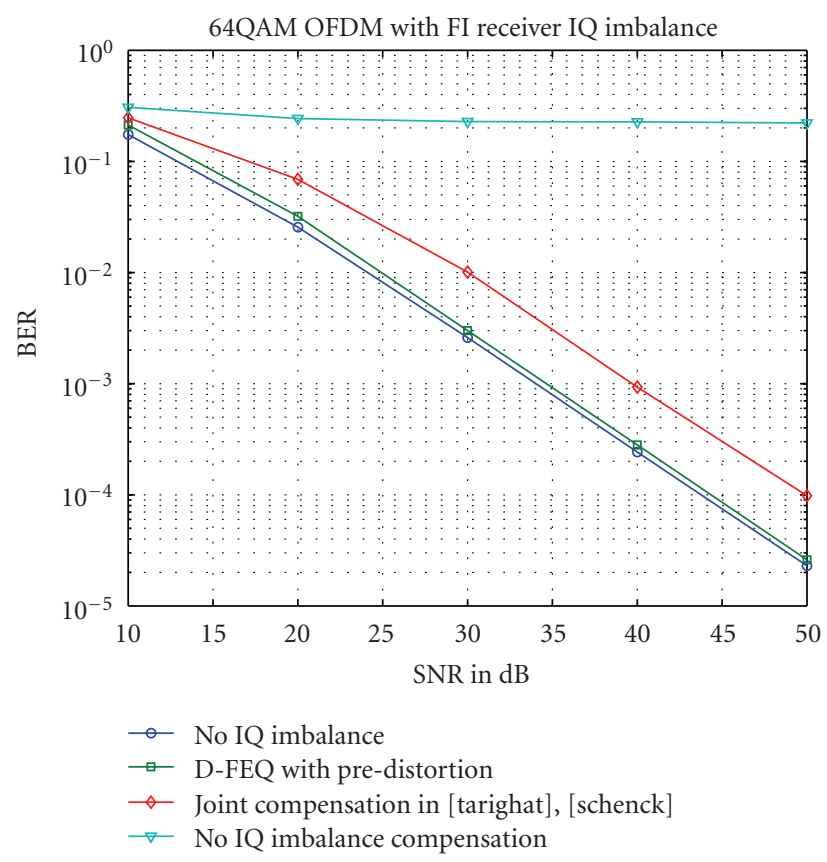

(b) BER versus SNR for receiver IQ imbalance

FIGURE 6: Performance results for 16QAM OFDM system with frequency independent amplitude imbalance of $g_{t}, g_{r}=5 \%$ and phase imbalance of $\phi_{t}, \phi_{r}=5^{\circ}$. The front-end filter impulse responses are $\mathbf{h}_{t i}=\mathbf{h}_{r i}=\mathbf{h}_{t q}=\mathbf{h}_{r q}=\left[\begin{array}{lll}0.01, & 0.5 & 0.06\end{array}\right]$. In the case of both transmitter/receiver IQ imbalance, D-FEQ with predistortion-based compensation scheme is implemented. (a) BER versus channel tap length at $20 \mathrm{~dB}$ SNR. (b) BER versus SNR performance results.

D-FEQ is very close to the ideal case, see Figure 5(d), thus typically extremely small amount of transmitter/receiver IQ imbalance can be safely ignored. In practice, the IRR $[l]$ due to IQ imbalance is in the order of $20-40 \mathrm{~dB}$ for one terminal (transmitter or receiver) [22]. The joint effect of transmitter and receiver IQ imbalance can thus expected to be more severe.

Figure 5(d) once again shows the BER versus SNR performance for a system impaired with transmitter and receiver IQ imbalance. It can be seen that the proposed predistortion-based D-FEQ compensation scheme is still very robust and the performance curves are very close to those of the ideal case even when only two LTS are used. The difference between the proposed scheme and the joint compensation scheme [19] is now almost $9 \mathrm{~dB}$ at BER of $10^{-3}$. Thus the proposed compensation scheme provides a very efficient compensation even with a very small training overhead.

Figures 6(a) and 6(b) consider the presence of FI IQ imbalance for 16QAM OFDM system, that is, we assume that the front-end filter impulse responses are perfectly matched $\mathbf{h}_{t i}=\mathbf{h}_{r i}=\mathbf{h}_{t q}=\mathbf{h}_{r q}=\left[\begin{array}{lll}0.01, & 0.5 & 0.06\end{array}\right]$. The transmitter and receiver frequency independent amplitude and phase imbalances are $g_{t}=g_{r}=5 \%$ and $\phi_{t}=\phi_{r}=5^{\circ}$, respectively. We once again consider $M_{l}=8$ LTS during the estimation phase of transmitter and receiver IQ imbalance gain parameters and then $M_{l}=2$ LTS during the estimation phase of only the channel characteristics. In Figure 6(a) we compare the
BER versus channel tap length (rms delay spread) at $20 \mathrm{~dB}$ SNR for D-FEQ scheme when both transmitter/receiver IQ imbalance are present, and when only receiver IQ imbalance is present. In the latter case, the transmitter IQ imbalance is considered perfectly matched. It can be observed from the figure that when the channel tap length is 1, that is, for a purely AWGN frequency flat channel, the D-FEQ scheme provides an effective compensation performance when only receiver IQ imbalance is considered in the system. Similar performance results will also be obtained for D-FEQ scheme when only transmitter IQ imbalance is considered. However, in the presence of both transmitter and receiver IQ imbalance, the D-FEQ scheme is not able to compensate as it requires frequency selectivity of the channel within the OFDM symbol in order to estimate the transmitter and receiver IQ imbalance gain parameters; see (32). When the channel tap length is greater than 1 , then the D-FEQ scheme exploits the frequency selectivity of the channel to obtain effective compensation performance. Thus, in the case of strict frequency flatness over the entire OFDM symbol, joint compensation scheme as shown in (15) should be performed.

Figure 6(b) once again shows the BER versus SNR performance for a 16QAM OFDM system impaired with FI transmitter and receiver IQ imbalance. The figure shows that the proposed D-FEQ scheme provides an efficient compensation performance with a very small training overhead requirement. 


\section{Conclusion}

In this paper, we have proposed training-based compensation schemes for OFDM systems impaired with transmitter and receiver IQ imbalance. The proposed schemes can decouple the compensation of the transmitter and receiver IQ imbalance from the compensation of the channel distortion. Once the IQ imbalance parameters are known, a standard channel equalizer can then be applied to estimate and compensate for channel variations in the system. The proposed schemes result in an overall lower training overhead and a lower computational requirement. Simulation results show that the proposed schemes provide a very efficient compensation with performance close to the ideal case without any IQ imbalance.

\section{Acknowledgments}

This research work was carried out at the ESAT Laboratory of Katholieke Universiteit Leuven and was funded in the framework of a DOC-DB scholarship of Katholieke Universiteit Leuven and the Belgian Programme on Inter-university Attraction Poles, initiated by the Belgian Federal Science Policy Office IUAP P6/04 (DYSCO, "Dynamical systems, control and optimization," 2007-2011). The scientific responsibility is assumed by its authors.

\section{References}

[1] J. A. C. Bingham, "Multicarrier modulation for data transmission: an idea whose time has come," IEEE Communications Magazine, vol. 28, no. 5, pp. 5-14, 1990.

[2] "IEEE standard 802.11a-1999: wireless LAN medium access control (MAC) \& physical layer (PHY) specifications, highspeed physical layer in the $5 \mathrm{GHz}$ band," 1999.

[3] I. Koffman and V. Roman, "Broadband wireless access solutions based on OFDM access in IEEE 802.16," IEEE Communications Magazine, vol. 40, no. 4, pp. 96-103, 2002.

[4] "ETSI Digital Video Broadcasting; Framing structure, Channel Coding \& Modulation for Digital TV," 2004.

[5] A. A. Abidi, "Direct-conversion radio transceivers for digital communications," IEEE Journal of Solid-State Circuits, vol. 30, no. 12, pp. 1399-1410, 1995.

[6] C.-L. Liu, "Impacts of I/Q imbalance on QPSK-OFDM-QAM detection," IEEE Transactions on Consumer Electronics, vol. 44, no. 8, pp. 984-989, 1998.

[7] S. Fouladifard and H. Shafiee, "Frequency offset estimation in OFDM systems in presence of IQ imbalance," in Proceedings of the International Conference on Communications (ICC '03), pp. 2071-2075, Anchorage, Alaska, USA, May 2003.

[8] J. Tubbax, B. Come, L. Van der Perre, M. Engels, M. Moonen, and H. D. Man, "Joint compensation of IQ imbalance and carrier frequency offset in OFDM systems," in Proceedings of the Radio and Wireless Conference, pp. 39-42, Boston, Mass, USA, August 2003.

[9] F. Horlin, A. Bourdoux, and L. Van Der Perre, "Lowcomplexity EM-based joint acquisition of the carrier frequency offset and IQ imbalance," IEEE Transactions on Wireless Communications, vol. 7, no. 6, Article ID 4543073, pp. 2212-2220, 2008.

[10] I. Barhumi and M. Moonen, "IQ-imbalance compensation for OFDM in the presence of IBI and carrier-frequency offset,"
IEEE Transactions on Signal Processing, vol. 55, no. 1, pp. 256266, 2007.

[11] A. Tarighat and A. H. Sayed, "Joint compensation of transmitter and receiver impairments in OFDM systems," IEEE Transactions on Wireless Communications, vol. 6, no. 1, pp. 240-247, 2007.

[12] D. Tandur and M. Moonen, "Joint adaptive compensation of transmitter and receiver IQ imbalance under carrier frequency offset in OFDM-based systems," IEEE Transactions on Signal Processing, vol. 55, no. 11, pp. 5246-5252, 2007.

[13] M. Valkama, M. Renfors, and K. Koivunen, "Compensation of frequency-selective IQ imbalances in wideband receivers: models and algorithms," in Proceedings IEEE 3rd Workshop on Signap Processing Advances in Wireless Communications (SPAWC '03), pp. 42-45, Taoyuan, Taiwan, March 2001.

[14] L. Anttila, M. Valkama, and M. Renfors, "Circularity-based I/Q imbalance compensation in wideband direct-conversion receivers," IEEE Transactions on Vehicular Technology, vol. 57, no. 4, pp. 2099-2113, 2008.

[15] L. Anttila, M. Valkama, and M. Renfors, "Efficient mitigation of frequency-selective I/Q imbalance in OFDM receivers," in Proceedings of the IEEE 68th Vehicular Technology Conference (VTC '08), Calgary, Canada, September 2008.

[16] E. Tsui and J. Lin, "Adaptive IQ imbalance correction for ofdm systems with frequency and timing offsets," in Proceedings of the IEEE Global Telecommunications Conference (GLOBECOM '04), pp. 4004-4010, Dallas, Tex, USA, November 2004.

[17] T. C. W. Schenk, P. F. M. Smulders, and E. R. Fledderus, "Estimation and compensation of frequency selective transmitter/receiver IQ imbalance in MIMO OFDM systems," in Proceedings of the IEEE International Conference on Communications (ICC '06), pp. 251-256, Istanbul, Turkey, July 2006.

[18] B. Narasimhan, D. Wang, S. Narayanan, H. Minn, and N. AlDhahir, "Digital compensation of frequency-dependent joint Tx/Rx I/Q imbalance in OFDM systems under high mobility," IEEE Journal on Selected Topics in Signal Processing, vol. 3, no. 3, pp. 405-417, 2009.

[19] D. Tandur and M. Moonen, "Joint compensation of OFDM frequency-selective transmitter and receiver IQ imbalance," Eurasip Journal on Wireless Communications and Networking, vol. 2007, Article ID 68563, 10 pages, 2007.

[20] D. Tandur, C.-Y. Lee, and M. Moonen, "Efficient compensation of RF impairments for OFDM systems," in Proceedings of the IEEE Wireless Communications and Networking Conference (WCNC '09), Budapest, Hungary, April 2009.

[21] K. Van Acker, G. Leus, M. Moonen, O. van de Wiel, and T. Pollet, "Per tone equalization for DMT-based systems," IEEE Transactions on Communications, vol. 49, no. 1, pp. 109-119, 2001.

[22] B. Razavi, "Design considerations for direct-conversion receivers," IEEE Transactions on Circuits and Systems II: Analog and Digital Signal Processing, vol. 44, no. 6, pp. 428-435, 1997. 